(C) Copyright 2010 American Meteorological Society (AMS). Permission to use figures, tables, and brief excerpts from this work in scientific and educational works is hereby granted provided that the source is acknowledged. Any use of material in this work that is determined to be "fair use” under Section 107 of the U.S. Copyright Act September 2010 or that satisfies the conditions specified in Section 108 of the U.S. Copyright Act (17 USC §108, as revised by P.L. 94-553) does not require the AMS's permission. Republication, systematic reproduction, posting in electronic form, such as on a web site or in a searchable database, or other uses of this material, except as exempted by the above statement, requires written permission or a license from the AMS. Additional details are provided in the AMS Copyright Policy, available on the AMS Web site located at (http://www.ametsoc.org/) or from the AMS at 617-227-2425 or copyright@ametsoc.org. 


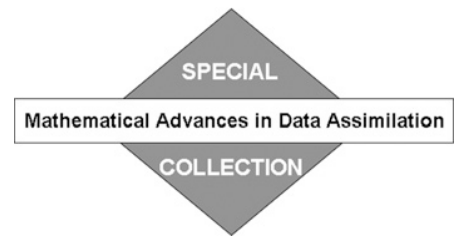

\title{
Robust Characterization of Model Physics Uncertainty for Simulations of Deep Moist Convection
}

\author{
DEREK J. POSSELT \\ University of Michigan, Ann Arbor, Michigan \\ TOMISLAVA VUKICEVIC \\ NOAA/Atlantic Oceanographic and Meteorological Laboratory, Miami, Florida
}

(Manuscript received 12 June 2009, in final form 6 November 2009)

\begin{abstract}
This study explores the functional relationship between model physics parameters and model output variables for the purpose of 1) characterizing the sensitivity of the simulation output to the model formulation and 2) understanding model uncertainty so that it can be properly accounted for in a data assimilation framework. A Markov chain Monte Carlo algorithm is employed to examine how changes in cloud microphysical parameters map to changes in output precipitation, liquid and ice water path, and radiative fluxes for an idealized deep convective squall line. Exploration of the joint probability density function (PDF) of parameters and model output state variables reveals a complex relationship between parameters and model output that changes dramatically as the system transitions from convective to stratiform. Persistent nonuniqueness in the parameter-state relationships is shown to be inherent in the construction of the cloud microphysical and radiation schemes and cannot be mitigated by reducing observation uncertainty. The results reinforce the importance of including uncertainty in model configuration in ensemble prediction and data assimilation, and they indicate that data assimilation efforts that include parameter estimation would benefit from including additional constraints based on known physical relationships between model physics parameters to render a unique solution.
\end{abstract}

\section{Introduction}

The increasing demand for accurate prediction of clouds and precipitation in numerical models at all scales has motivated the implementation of increasingly realistic cloud and precipitation microphysical parameterizations. These are typically bulk schemes, which are based on the assumption that the particle size distribution for each form of liquid and ice condensate takes a particular form. The simplifying assumptions about the form of the particle size distribution of ice and liquid condensate, which require a priori specification of parameter values, have an important effect on the details of cloud and precipitation development. It has been shown that, regardless of the details of the microphysical formulation, model output is nearly uniformly highly sensitive to small changes in these parameters (Fovell and Ogura 1989;

Corresponding author address: Derek J. Posselt, University of Michigan, 2455 Hayward St., Ann Arbor, MI 48109-2143. E-mail: dposselt@umich.edu
Ferrier 1994; Walko et al. 1995; Ferrier et al. 1996; Meyers et al. 1997; Grabowski et al. 1999; Grabowski 2003). Traditional sensitivity experiments consist of defining a control simulation, perturbing a given parameter or set of parameters, rerunning the model, and comparing the results with the control. Several cloud-system-resolving model (CRM) sensitivity experiments have been conducted in this manner (Tao et al. 1995; Walko et al. 1995; Meyers et al. 1997; Grabowski et al. 1999; Wu et al. 1999; Petch and Gray 2001; Grabowski 2003; Saleeby and Cotton 2004; Gilmore et al. 2004; van den Heever and Cotton 2004), and have shown that CRM-simulated cloud fields are particularly sensitive to changes in the parameters that define particle size distributions.

A number of recent studies have used data assimilation techniques to study the effects of variation in model physics parameters (Aksoy et al. 2006; Tong and Xue 2008) or parameterization schemes (Meng and Zhang 2007). Instead of seeking to understand the nature of the uncertainty, the primary goal of these data assimilation 
experiments has been to account for it as a source of model error in the assimilation system and to produce a more accurate estimate of the state of the atmosphere. The principle that underlies sensitivity studies and data assimilation experiments is the fact that a functional relationship exists between parameters and model state. The extent to which this relationship can be quantified has been termed "parameter identifiability" in the ensemble data assimilation literature (Anderson 2001; Aksoy et al. 2006; Tong and Xue 2008). Regardless of whether the ultimate goal is to understand or to mitigate model uncertainty, two key pieces of information are needed: the functional relationship between parameters and how changes in parameter values map to changes in model output. Both pieces can be obtained by characterizing the space that results from a combination of 1) information about the set of physically realistic parameter values, 2) the associated output from a model of the system, and 3) the range of possible measurement values. This information can be quantified by assigning a probability distribution to each piece of information; the joint probability density function (PDF) that results from the conjunction of these probability distributions defines both the sensitivity and the data assimilation problem in the context of inverse problem theory (Vukicevic and Posselt 2008, hereafter VP08). The challenge is to construct the inverse problem in such a way that this joint PDF can be quantified. If this PDF is available, it can be used to examine which parameters have the most significant influence on the model, the relationships between parameters, and which model output variables are most sensitive to changes in the parameters. The resulting information can then be used to determine how to best perturb parameters in an ensemble forecasting or assimilation context.

Ensemble Kalman filter-type data assimilation methods address this problem by assuming each space is characterized by a Gaussian probability distribution and attempting to span (sample) the covariance of the analysis using an ensemble of model simulations. However, the assumption of Gaussian statistics can be problematic if the relationship between control variables and observations is nonmonotonic nonlinear (VP08). This is reflected in the fact that parameter identifiability has been shown to decrease when the numbers of parameters estimated in ensemble data assimilation increases. Aksoy et al. (2006) and Tong and Xue (2008) showed that, although a unique solution could be obtained when individual parameters were estimated, when multiple parameters were included in the assimilation the estimation of parameter values became much less reliable.

In contrast to methods that rely on the assumption of Gaussian statistics, Markov chain Monte Carlo (MCMC) algorithms have been shown to robustly characterize the solution space and to flexibly incorporate changes to the assumptions of the nature of uncertainty in observations and the model (Posselt et al. 2008; Tamminen 2004). In this study, we use an MCMC algorithm to characterize the nature of the uncertainty in simulations of convective clouds and precipitation associated with the specification of cloud microphysical parameters in a Lagrangian cloud model. We use a single-moment bulk microphysical scheme similar to those used in modern regional and global numerical prediction and climate models and examine a case of squall-line-type convection, which is known to have large sensitivity to changes in cloud microphysical parameters. The MCMC algorithm reveals a nonmonotonic relationship between parameters and model output that gives rise to a multimode parameter space. The examination of joint parameter-state PDFs indicates that this relationship changes with time over the course of the simulation. The addition of information in the form of additional observations or reduction of observation errors serves to eliminate many of the possible modes in the parameter space, but it cannot fully eliminate the occurrence of a nonunique solution, which is tied to nonuniqueness in the model formulation itself. We demonstrate that knowledge of the presence of nonmonotonic nonlinearity in the parameter-state relationships, along with an understanding of the physical nature of the system, can be used to adjust the inverse problem so that a one-to-one mapping between parameters and state can be achieved.

The remainder of this paper is organized as follows: Section 2 contains a description of the model framework and information on the control parameters and observations used in the sensitivity analysis. We outline the general inverse method in section 3 and describe how a Markov chain Monte Carlo algorithm can be used to sample the posterior joint probability distribution. We present the results of running the MCMC algorithm on the cloud model in section 4 and discuss caveats and implications. In section 5, we offer a summary of our results and our primary conclusions, present a brief discussion of the limitations of our study, and outline our anticipated future work.

\section{Modeling framework}

Uncertainty in cloud microphysical parameters affects simulated cloud and precipitation fields, which naturally feed back to the dynamics on multiple scales. Because of the complexity in the microphysics-dynamics relationship, a necessary first step in understanding the details of model error is to consider the effect of changes in model physics parameters on the model output in isolation from any feedback to the cloud-scale dynamics. Such 
an exercise requires a framework that accommodates realistic changes to the environment so that the cloud microphysical routine can respond as if it were embedded in a fully interactive model. To do this, we have constructed what we term a "Lagrangian column model," which is designed to emulate the changes in environment experienced by an atmospheric column as it moves through a cloud system following the mean flow. The model consists of a single-column version of the National Aeronautics and Space Administration (NASA) Goddard Cumulus Ensemble (GCE) model (Tao and Simpson 1993; Tao et al. 2003). As in the fully threedimensional version of the GCE, base-state vertical profiles of potential temperature and moisture are fixed and the model predicts perturbations on these base-state profiles. In our idealized framework, clouds are generated by forcing the model with time-varying vertical profiles of vertical motion and water vapor tendency. Advection is only allowed to operate on cloud liquid and ice condensate and only in the vertical direction. By specifying appropriate base-state potential temperature and water vapor and time-varying vertical profiles of vertical motion and water vapor forcing, the model can be used to simulate the flow through a range of different cloud systems.

Because organized deep convection produces the bulk of the warm-season precipitation globally, and has been shown to be highly sensitive to changes in cloud microphysical parameters, we use as our test case an idealized representation of squall-line-type convection. The added benefit to the examination of squall-line-type convection is that it contains two discrete cloud morphologies: convective, in which precipitation is primarily generated by the collision-coalescence (warm rain) process; and stratiform, in which the melting of snow and graupel play a key role. The simulation is constructed so that it is consistent with idealized (Moncrieff 1992) and observed (Houze 2004) squall lines. Profiles are assumed to be sinusoidal with the following functional forms for vertical motion:

$$
w(z, t)= \begin{cases}w_{0}(t) \cos ^{4}\left\{\frac{\pi}{2} \frac{\left[z-z_{0 w}(t)\right]}{D_{w}(t)}\right\}, & \left|z-z_{0 w}(t)\right| \leq D_{w}(t) \\ 0, & \left|z-z_{0 w}(t)\right|>D_{w}(t)\end{cases}
$$

and for water vapor tendency

$$
q^{\prime}(z, t)= \begin{cases}q_{0}(t) \cos ^{2}\left\{\frac{\pi}{2} \frac{\left[z-z_{0 q}(t)\right]}{D_{q}(t)}\right\}, & \left|z-z_{0 q}(t)\right| \leq D_{q}(t) \\ 0, & \left|z-z_{0 q}(t)\right|>D_{q}(t)\end{cases}
$$

Use of a sinusoidal function allows specification of full profiles of vertical motion and water vapor tendency using only three parameters: the maximum value $w_{0}$ and $q_{0}$, height of the maximum value $z_{0 w}$ and $z_{0 q}$, and sine function half-width $D_{w}$ and $D_{q}$-all of which are allowed to vary in time. The resulting profiles (Fig. 1) bear a close resemblance to the observed vertical distribution of latent heating and vertical motion in tropical squall lines (Houze 2004 and references therein). In our idealized squall-line simulation, the column enters the system at the leading edge of the cold pool, propagates through the convective precipitation region, and exits through the stratiform precipitation region. Base-state profiles of temperature and water vapor are representative of the tropical oceanic environment (Fig. 1a) and are generated by setting surface temperature and water vapor mixing ratio equal to values typical of the tropical Pacific warm-pool region, then decreasing temperature at a pseudo- (moist) adiabatic lapse rate. Water vapor mixing ratio $q_{v}$ is held constant with height until the atmosphere reaches liquid saturation, after which point $q_{v}$ decreases at a rate that maintains saturation with respect to liquid at temperatures above freezing and with respect to ice below. Time-evolving profiles of vertical motion and water vapor forcing (Figs. 1b,c, respectively) consistent with observed and simulated squall-line-type evolution (Moncreiff 1992; Fovell and Tan 1998; Houze 2004) are used to drive the simulation. The model is run with 60 vertical layers with constant 250 -m vertical grid spacing and a 5-s time step, and the radiative transfer, surface flux, and microphysical parameterizations are all identical to those used in the NASA Goddard Cumulus Ensemble model (Tao and Simpson 1993; Tao et al. 2003; Lang et al. 2007). The time series of a number of model output variables are shown in Figs. 2a-e, and it can be seen that the model produces realistic time evolution of precipitation rate (PCP), liquid and ice water path (LWP and IWP, respectively) and top of the atmosphere shortwave (SW) and longwave (LW) radiative fluxes [outgoing SW radiation (OSR) and outgoing LW radiation (OLR)]. Time-height cross sections of mass mixing ratio for each condensate species are plotted in 

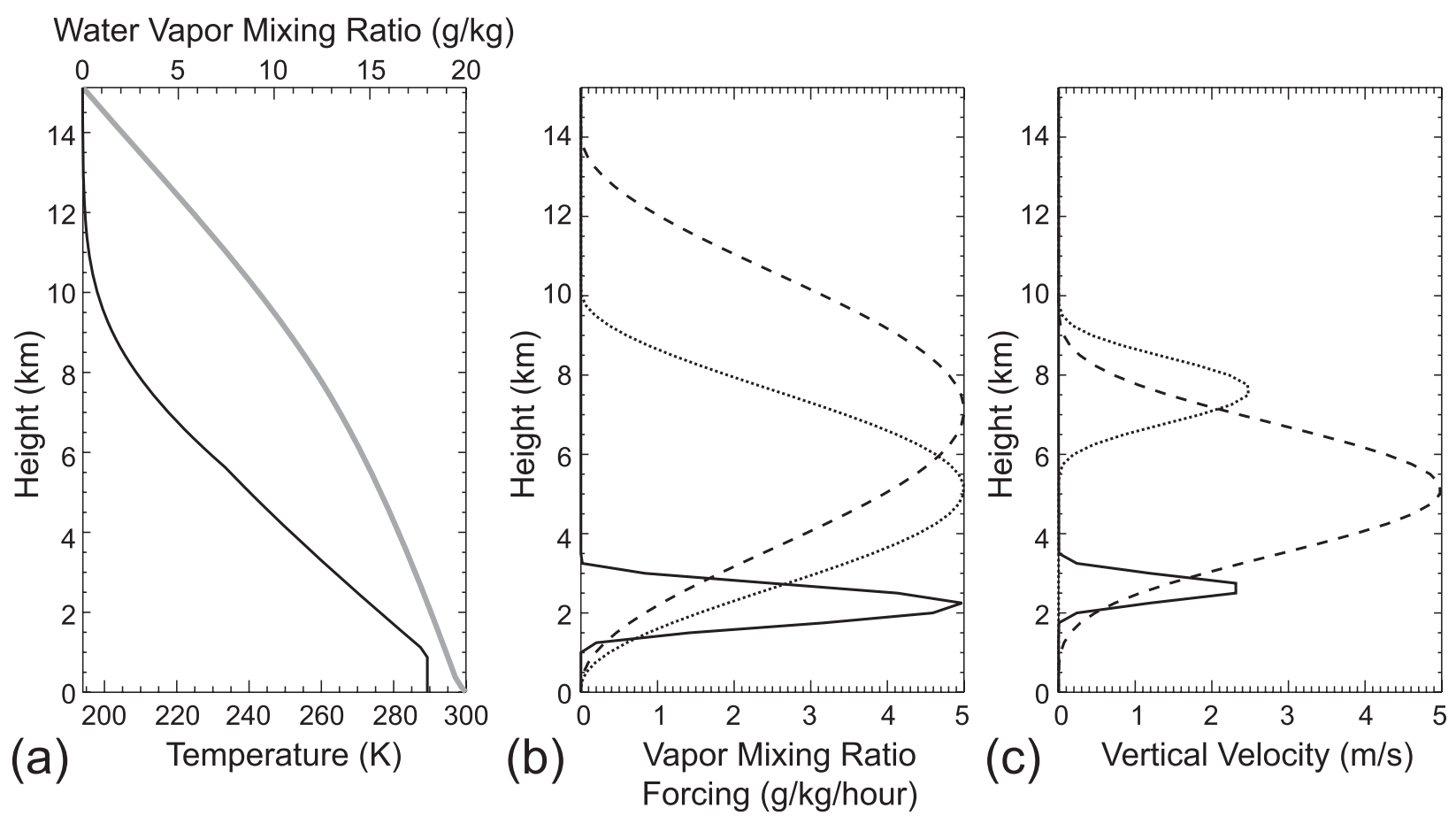

FIG. 1. Profiles of (a) temperature (solid black line) and water vapor (solid gray line), (b) initial- and time-evolving profiles of water vapor forcing, and (c) initial- and time-evolving profiles of vertical motion. In (b) and (c), the solid line corresponds to the initial time, the dashed line to $60 \mathrm{~min}$ of simulation time, and the dotted line to $120 \mathrm{~min}$ of simulation time.

Figs. 2f-j; convective and stratiform regions of precipitation are clearly discernible.

Because this paper seeks to characterize model uncertainty associated with the specification of bulk microphysical parameters, we offer a brief description of the cloud microphysical parameterization here. The GCE model employs a single-moment bulk scheme, in which two classes of liquid (rain and nonprecipitating cloud) and three classes of ice (nonprecipitating pristine crystals, precipitating unrimed pristine crystals and aggregates, and graupel) are assumed to follow an exponential particle size distribution (Lin et al. 1983; Rutledge and Hobbs 1983; 1984; Tao et al. 2003; Lang et al. 2007) that, for cloud species $x$, takes the form

$$
N_{x}(D)=N_{0 x} \exp \left(-\lambda_{x} D\right)
$$

Here, $N_{0 x}$ is the slope intercept and $\lambda_{x}$ is the slope,

$$
\lambda=\left(\frac{\pi \rho_{x} N_{0 x}}{\rho q_{x}}\right)^{1 / 4},
$$

where $\rho_{x}$ is the condensate density, $\rho$ is the air density, and $q_{x}$ is the condensate mass mixing ratio. In addition to the specification of the slope intercept of the particle size distribution and the ice particle density (rain is assumed to have a constant density equal to liquid water at $0^{\circ} \mathrm{C}$ ), the particle fall speed is parameterized using a power relationship,

$$
V_{x}(D)=a_{x} D^{b_{x}},
$$

where the values assigned to the coefficient $a_{x}$ and exponent $b_{x}$ depend on the assumed ice crystal shape and are typically taken from in situ observations of precipitating ice (Locatelli and Hobbs 1974; Mitchell 1996; Heymsfield et al. 2002). Though this single-moment scheme is relatively simple compared with modern twoand three-moment and bin schemes, it is representative of the complexity of microphysical parameterizations that are currently used in regional prediction models and quite a bit more sophisticated than those used in GCMs, which typically use one species of liquid and ice as prognostic variables and diagnose the partition between suspended and precipitating condensate.

Our goals in this study are to use the model framework outlined earlier to explore the sensitivity of simulation output to changes in model physics assumptions and to characterize the degree of nonlinearity present in the parameter-state relationship. To this end, we examine the influence of changes to cloud microphysical parameters on simulated PCP, LWP, IWP, OSR, and OLR, as they are directly related to cloud microphysical processes relevant to the study of cloud radiative feedbacks in the 

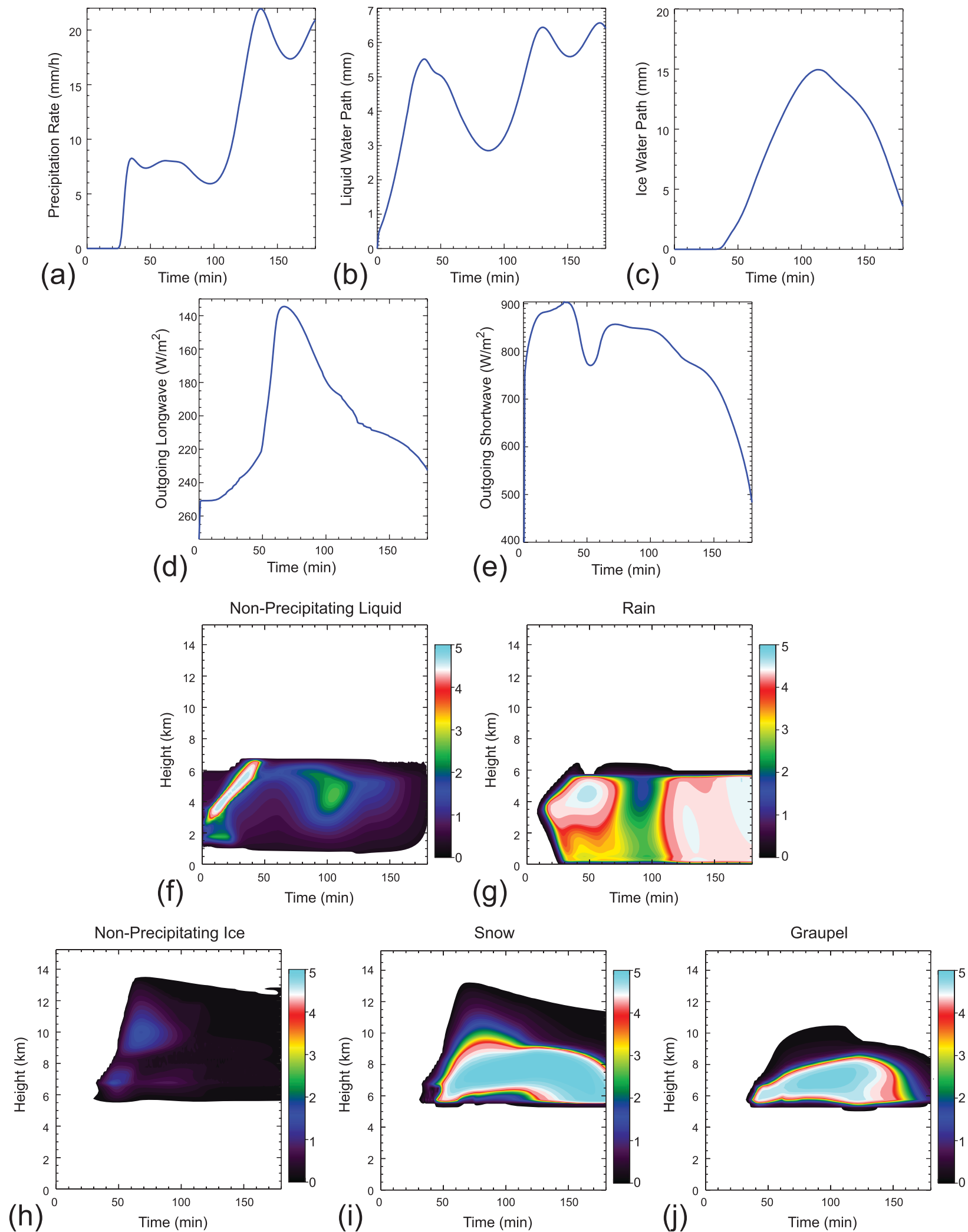

FIG. 2. Plots of (a)-(e) model output variables vs time and (f)-(j) time-height cross sections of condensate variables. 
climate system and are available as retrievals from current satellite remote sensing platforms. These variables also serve as observations in our inverse modeling algorithm, and we note that because they are output directly from the model, we require no observation operator. Avoiding the use of a complicated (and likely nonlinear) observation operator (as would be necessary for radar reflectivity observations, for example) allows for a cleaner evaluation of the relationship between changes to parameters and the effect on model output. We note the pros and cons of our choice of observations later in section 5 .

Thorough examination of parameter-state relationships necessitates perturbation of all parameters simultaneously; it is the relationships between parameters and their joint effect on model output that is of interest. Because this is a computationally demanding exercise, it is useful to briefly explore the model sensitivity in a more simplistic way before we begin to ensure that the model is appropriately sensitive to changes in cloud microphysical parameters. To this end, we run two experiments in which we examine the sensitivity of precipitation rate, LWP, IWP, OLR, and OSR at $120 \mathrm{~min}$ of simulation time to systematic changes in the values of the graupel fall speed coefficient and the graupel density (Fig. 3). These plots are analogous to Figs. $1 b$ and $2 b$ in VP08, and they effectively map the functional relationship between the chosen microphysical parameter and the model output. It can be seen that the model is sensitive to changes in each of these parameters; model output varies widely across the range of parameter values. The graupel fall speed coefficient exhibits nonmonotonic nonlinearity; multiple values of this parameter produce identical values of each of the model output variables. The graupel density exhibits a nonlinear relationship with each of the output variables, however, the nature of the relationship is monotonic; each value of graupel density maps to a single value of each of the model output variables. The primary conclusions that arise from an examination of Figs. 2 and 3 are that the model 1) produces a realistic simulation that is sensitive to changes in microphysical parameters, 2) exhibits nonlinear relationships between parameters and state, and 3) includes two distinct cloud microphysical regimes.

\section{Inverse problem framework}

Model uncertainty can be quantified using the framework of inverse problem theory, as postulated in Mosegaard and Tarantola (2002). The most general solution to an inverse problem consists of the conjunction of the various information spaces that define the state of knowledge of the system (Rodgers 2000; Mosegaard and
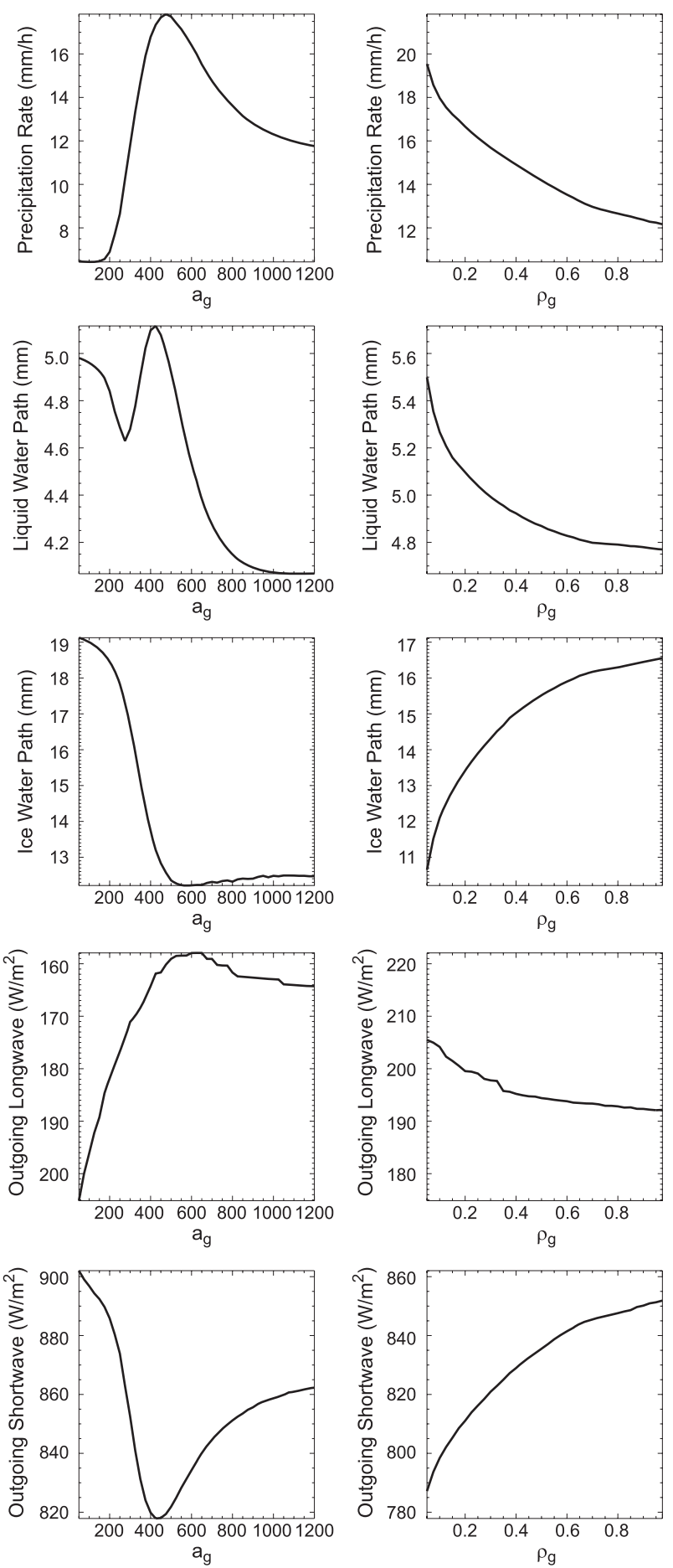

FIG. 3. Plots of changes in (left) graupel fall speed coefficient and (right) graupel density vs precipitation rate (first row), LWP (second row), IWP (third row), OLR (fourth row), and OSR (fifth row). Model output is observed at 120 min into the simulation. 
Tarantola 2002; Tarantola 2005; VP08). The character and magnitude of the uncertainties in observations and forward model translate directly to the extent and shape of their corresponding information spaces, and the uncertainty in the resulting estimate is similarly characterized by the extent and shape of the solution information space. If uncertainties in measurements, model, and prior estimates are represented by probability distributions, then the solution is defined as the joint posterior distribution of the observations and control parameters. The optimal parameter estimate is, by definition, the location in the joint distribution $P(\mathbf{x}, \mathbf{y})$ with the greatest probability density, and the error characteristics for each control parameter can be examined by considering each parameter's marginal probability distribution. Similarly, the relationship between parameters can be examined by considering their joint distribution, whereas the functional relationship between parameters and state is expressed in the joint distribution of parameters and model output state. The marginal probability distribution for each parameter, subset of parameters, or parameter observation pair is computed by integrating the joint PDF over all parameters except the parameter[(s) and observation(s)] of interest. The ensemble Kalman filter and variational data assimilation methods are specific types of solution to the general inverse problem (VP08), but the fundamental strength of the information-based framework lies in its generality. It treats contributions from the model, model error, observations, and prior state as probability distributions with no assumed form and characterizes the solution as the joint posterior probability distribution that results from conjunction of the component PDFs.

Following VP08, the definition of conditional probability can be used to write the joint PDF of model parameters $\mathbf{x}$ and observations $\mathbf{y}$ in the familiar Bayes theorem form

$$
P(\mathbf{x} \mid \mathbf{y})=\frac{P(\mathbf{y} \mid \mathbf{x}) P(\mathbf{x})}{P(\mathbf{y})},
$$

where $P(\mathbf{x} \mid \mathbf{y})$ is the posterior probability density to be solved for, $P(\mathbf{x})$ is the prior probability density of the set of parameters $\mathbf{x}, P(\mathbf{y} \mid \mathbf{x})$ is the probability density that describes the likelihood that the parameters are the true parameters (given the uncertainty in the model and observations), and $P(\mathbf{y})$ is the prior probability density of the observations. The optimal parameter estimate is, by definition, the maximum likelihood point in the conditional distribution $P(\mathbf{x} \mid \mathbf{y})$, and the error characteristics for the set of control parameters can be examined by considering the properties (e.g., moments) of the conditional PDF. Note that we have made no assumptions about the form of the probability distributions in (6), hence the complete information on the parameter interaction and parameter-state functional relationship is present in the solution to this formulation of the generalized inverse problem.

It remains, then, to find a way to compute the solution to (6). Most modern data assimilation techniques and retrieval algorithms are based on the assumption that probabilities are Gaussian. The derivation of the solution to (6) for the case of Gaussian PDFs has been well documented (Rodgers 2000; Kalnay 2003; Evensen 2006; Lewis et al. 2006; VP08; among many others) and will not be repeated here. Instead, we address the question of how to compute each PDF in the case where PDFs are not assumed to be Gaussian, and, in fact, may not have a unique mode. Perhaps the most direct method of solution is to resolve each of the parameters of interest into discrete bins across their ranges of natural variability, assign a form and magnitude for the measurement uncertainty, and convolve the resulting PDFs via numerical computation. Such an exercise formed the basis for the algorithm described in VP08; however, the computational expense of doing so increases as $N^{M}$, where $N$ is the desired resolution of the parameter space and $M$ is the number of parameters allowed to vary. For joint probability distributions that contain a moderate amount of mass in each dimension, the theory of empty spaces (Tarantola 2005) dictates that the amount of empty space in the solution space (e.g., the percentage of the space for which values of the parameters are associated with very low probability) tends to increase exponentially with each additional dimension. It follows that an effective method of characterizing the probability density function is one that avoids empty (low probability) regions of the space in favor of regions with relatively high probability density, where high-density regions are identified via a predefined measure of fit between simulation output and observations (e.g., cost function). Markov chain Monte Carlo algorithms comprise a class of such methods. Markov chain Monte Carlo methods address the problem of empty parameter spaces by sampling the probability distribution in such a way as to revisit those regions with high probability while avoiding regions with low probability (Tamminen and Kyrola 2001, hereafter TK01; Tarantola 2005; Posselt et al. 2008). Specifically, the MCMC algorithm is made up of a random walk that consists of multiple successive iterations (see flowchart in Fig. 4). In each iteration, test values $\tilde{\mathbf{x}}$ of all parameters are randomly drawn from a "proposal" distribution $\left\{\tilde{\mathbf{x}} \sim q\left[\tilde{\mathbf{x}} ; \mathbf{x}^{(i)}\right]\right\}$ that depends on the current estimate $\mathbf{x}^{(i)}$. Proposed parameter values are then used in the forward model, and the resulting simulated measurements $[\tilde{\mathbf{y}}=f(\tilde{\mathbf{x}})]$ and prior $[p(\tilde{\mathbf{x}})]$ are compared with 


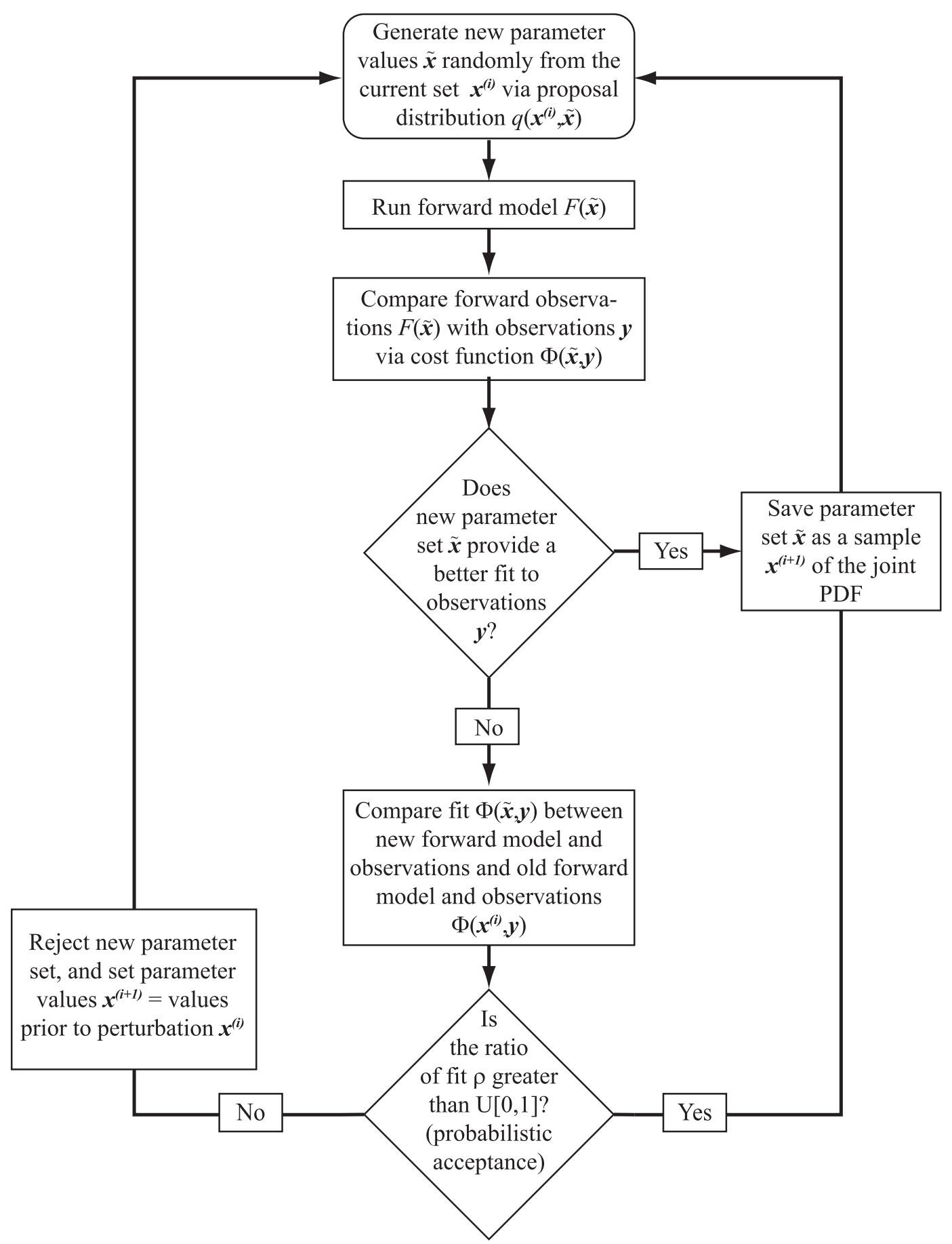

FIG. 4. Depiction of the order of operations in each MCMC iteration.

observations via a cost function derived from the specified form of $P(\mathbf{y} \mid \mathbf{x})$. The proposed parameter values are accepted as a sample in the posterior distribution with the probability

$$
Q\left[\tilde{\mathbf{x}} ; \mathbf{x}^{(i)}\right]=\min (\rho, 1),
$$

where the acceptance ratio $\rho$ is defined as

$$
\rho=\frac{P(\tilde{\mathbf{x}}) P(\mathbf{y} \mid \tilde{\mathbf{x}}) q\left[\tilde{\mathbf{x}} ; \mathbf{x}^{(i)}\right]}{P\left[\mathbf{x}^{(i)}\right] P\left[\mathbf{y} \mid \mathbf{x}^{(i)}\right] q\left[\mathbf{x}^{(i)} ; \tilde{\mathbf{x}}\right]} .
$$

If the prior parameter probability distribution is con(7) stant (e.g., uniform), and the proposal distribution is 
symmetric $\left\{\right.$ e.g., $\left.q\left[\tilde{\mathbf{x}} ; \mathbf{x}^{(i)}\right]=q\left[\mathbf{x}^{(i)} ; \tilde{\mathbf{x}}\right]\right\}$, then the acceptance ratio reduces to

$$
\rho=\frac{P(\mathbf{y} \mid \tilde{\mathbf{x}})}{P\left[\mathbf{y} \mid \mathbf{x}^{(i)}\right]},
$$

which can easily be computed from the specified form of the observation uncertainty PDF applied to the current and proposed parameter values. Note that this also eliminates the need for an a priori term in the cost function.

The value of the acceptance ratio determines whether the proposed set of parameters is saved as a sample of the posterior distribution. If the new parameters generate a better fit to observations $(\rho>1)$, then the proposed set of parameters is saved as the next sample in the distribution $\left[\mathbf{x}^{(i+1)}=\tilde{\mathbf{x}}\right]$. If not $(\rho<1)$, then a test value is drawn from a uniform $(0,1)$ distribution. If this value is less than $\rho$, then the proposed parameter set is also accepted as a sample in the distribution. Otherwise, the proposed set of parameters is rejected, and new proposed parameter values are drawn based on the original set. The accept/ reject procedure ensures that parameter sets that provide a better fit to the observations are immediately accepted, those that provide a similar fit are considered, and those that lead to simulated observations that are very different from the measurements are rejected. In the process, regions of the parameter space with relatively high probability are preferentially sampled, whereas regions with low probability are avoided, and a sample of the posterior distribution is produced using far fewer iterations than direct computation of the PDF. A more complete description of the theoretical underpinnings of the MCMC algorithm can be found in Mosegaard and Tarantola (2002) and in Tarantola (2005), whereas tools for assessing convergence of MCMC to sampling a stationary distribution are discussed in Gelman et al. (1996, 2004).

It should be noted that, in the process of sampling the joint parameter-observation information space, MCMC identically returns a solution to Bayes theorem (6). The computation of the cost function (measure of fit between model state and observations) for the set of MCMC iterations is identical to the computation of $P(\mathbf{y} \mid \mathbf{x})$, and the parameter values generated over multiple MCMC iterations is a sample of $P(\mathbf{x} \mid \mathbf{y})$. The application to parameter sensitivity follows from the fact that, in sampling the joint parameter PDF, MCMC effectively returns a nonlinear estimate of the parameter-state sensitivity (Jacobian) matrix. However, in contrast to linear estimates of sensitivity, the PDF output from MCMC contains a description of the full functional relationship between parameters and model output given the true model parameters. As such, the sample of the PDF returned by the MCMC algorithm can be used to characterize the relationship between parameters and model state, and it allows the sources of error that dominate the uncertainty in the result to be robustly quantified. Testing different error assumptions, including specification of not only different error magnitudes but also different error probability distributions, is straightforward, as is the introduction of new observations and the evaluation of new physical models.

Compared with methods that are constrained to particular forms of the PDFs in (6), the design of an MCMC algorithm is highly flexible, and the choice of prior estimate of control parameters $P(\mathbf{x})$ and the magnitude and form of the observation uncertainty $P(\mathbf{y} \mid \mathbf{x})$ can be adapted to suit the specific inverse problem. Our implementation of the MCMC algorithm is documented in Posselt et al. (2008) and is based on the work of TK01), who employed a Gaussian proposal distribution centered on the current parameter estimate. However, in contrast to the technique used in TK01, we do not use the covariance between parameters to generate parameter perturbations. In the case of a unimodal joint parameter distribution, generating parameter perturbations from a sample of the parameter covariance can improve the efficiency of the MCMC algorithm. However, if the parameter joint PDF is multimodal (e.g., the functional relationship between parameters and observations is nonmonotonic), parameter-parameter covariance can change sign for different combinations of parameter values. Because parameter covariance in the TK01 algorithm is updated infrequently, nonmonotonicity can lead to parameter perturbations that are based on an improper covariance estimate and to an inability to effectively sample the parameter space. Consequently, we have chosen to apply uncorrelated Gaussian perturbations to our set of control parameters. It should also be noted that, if perturbations to parameters are too large, the algorithm will not sample the PDF with sufficient resolution; however, if perturbations are too small, the algorithm will be inefficient and may not visit all regions of the space. For this reason, the variance of the proposal distribution is adaptively changed during a burn-in period to give the desired sampling rate. Samples of the posterior PDF obtained during burn in are not included in the final analysis. For a discussion on optimal sampling rates, the reader is referred to Gelman et al. (1996) and Haario et al. (1999).

We choose as control variables 10 cloud microphysical parameters known to have a significant influence on simulated clouds, precipitation, and radiative fluxes. The set of chosen parameters, along with their units and range of variability, are detailed in Table 1 . We assign a bounded uniform PDF as the prior parameter distribution and set the parameter ranges to correspond to observations of ice particle fall speeds (Locatelli and Hobbs 1974; Mitchell 1996) and liquid and ice particle size distributions (Tokay 
TABLE 1. Cloud microphysical parameters used in the MCMC algorithm, along with truth values for the simulated observation experiment and parameter ranges. Note that we have reported all values in cgs units to be consistent with what is used in the model formulation and inverse method.

\begin{tabular}{|c|c|c|c|c|c|}
\hline Parameter description & Abbreviation & Units & Truth & Min & $\operatorname{Max}$ \\
\hline Snow fall speed coefficient & $a_{s}$ & $\mathrm{~cm}^{1-b_{s}}$ & 200.0 & 50.0 & 1000.0 \\
\hline Snow fall speed exponent & $b_{s}$ & none & 0.3 & 0.1 & 1.0 \\
\hline Graupel fall speed coefficient & $a_{g}$ & $\mathrm{~cm}^{1-b_{g}}$ & 400.0 & 50.0 & 1200.0 \\
\hline Graupel fall speed exponent & $b_{g}$ & none & 0.4 & 0.1 & 0.9 \\
\hline Slope intercept of the rain particle size distribution & $N_{0 r}$ & $\mathrm{~cm}^{-4}$ & 0.5 & 0.0 & 5.0 \\
\hline Slope intercept of the snow particle size distribution & $N_{0 s}$ & $\mathrm{~cm}^{-4}$ & 0.5 & 0.0 & 5.0 \\
\hline Slope intercept of the graupel particle size distribution & $N_{0 g}$ & $\mathrm{~cm}^{-4}$ & 0.5 & 0.0 & 5.0 \\
\hline Snow particle density & $\rho_{s}$ & $\mathrm{~g} \mathrm{~cm}^{-3}$ & 0.2 & 0.1 & 1.0 \\
\hline Graupel particle density & $\rho_{g}$ & $\mathrm{~g} \mathrm{~cm}^{-3}$ & 0.4 & 0.1 & 1.0 \\
\hline Threshold cloud mass mixing ratio for autoconversion to rain & $q_{c_{0}}$ & $\mathrm{~g} \mathrm{~kg}^{-1}$ & 1.0 & 0.1 & 3.0 \\
\hline
\end{tabular}

and Short 1996; Roy et al. 2005; Heymsfield et al. 2002). In specifying a bounded uniform as the parameter prior, we make the prior distribution nearly noninformative, though there is some potential dependence on the specified parameter range. We tested a variety of ranges for parameter values and found that the PDF returned by MCMC is insensitive to the exact range of parameter values used in the algorithm, as long as it spans the range variability observed in nature.

In each experiment, a truth state is generated by running the model with parameter values consistent with what is currently used in cloud-resolving and mesoscale models (Table 1). Observations are drawn from this truth state, and observation errors are assumed to have a Gaussian form, with variance consistent with the rigorous quantification of uncertainty performed on the analogous retrieved quantities from the Tropical Rainfall Measuring Mission (TRMM; L'Ecuyer and Stephens 2002, 2003). It should be noted that the form of the observation error is not constrained to be Gaussian (Posselt et al. 2008); we use this form in our experiments to be consistent with the error estimates associated with TRMM retrievals. Observation uncertainty standard deviations are listed in Table 2, and the truth state from which observations are drawn is plotted in Figs. 2a-e.

Convergence of the MCMC algorithm to sampling a stationary distribution is assessed by comparing distributions generated by drawing random sets of parameters from the full sample. According to the results presented by Haario et al. (1999), between 20000 and 40000 iterations should be sufficient to sample a 10-dimensional parameter space if the target (posterior) distribution is multivariate Gaussian. In reality, as was indicated in the preliminary results presented in Fig. 3, the underlying PDF is non-Gaussian, and we find that approximately 100000 iterations are necessary to sample the underlying PDF. To ensure a robust sample, we ran MCMC out to $4 \times 10^{6}$ iterations. Convergence to sampling a stationary distribution was ensured by drawing random samples of 100000 parameter sets from the full MCMC sample and computing the integrated absolute difference between histograms. We found an average of less than 1\% difference between any two given random samples, indicating robust sampling of the underlying (true) PDF. To illustrate convergence of the MCMC algorithm to sampling a stationary distribution, we have plotted the joint PDF of two of the model parameters for successive numbers of iterations (Fig. 5). As early as 5000 iterations, the shape of the underlying PDF is beginning to emerge, and by 50000 iterations, the characteristics of this particular joint PDF appear to be stationary. Recall that 10 parameters are varied in this example; direct numerical computation of the PDF (assuming a resolution of 10 bins per parameter) would require $10^{10}$ simulations; MCMC returns a robust sample of the PDF using fewer than $10^{6}$ simulations.

\section{Probabilistic assessment of model physics uncertainty}

\section{a. Sensitivity of clouds and precipitation to model error}

Having introduced the model and inverse problem framework, we now assess the sensitivity of simulated clouds and precipitation to changes in microphysics parameters. To this end, we run MCMC using the set of 10

TABLE 2. Observations used in the MCMC parameter sensitivity experiment, along with their units and error estimates.

\begin{tabular}{cll}
\hline \hline Observation & \multicolumn{1}{c}{ Units } & \multicolumn{1}{c}{ Error } \\
\hline PCP & $\mathrm{mm} \mathrm{h}^{-1}$ & $2.0 \mathrm{~mm} \mathrm{~h}^{-1}$ \\
LWP & $\mathrm{mm}$ & $0.5 \mathrm{~mm}$ \\
IWP & $\mathrm{mm}$ & $1.0 \mathrm{~mm}$ \\
OLR & $\mathrm{W} \mathrm{m}^{-2}$ & $10 \mathrm{~W} \mathrm{~m}$ \\
OSR & $\mathrm{W} \mathrm{m}^{-2}$ & $20 \mathrm{~W} \mathrm{~m}^{-2}$ \\
\hline
\end{tabular}



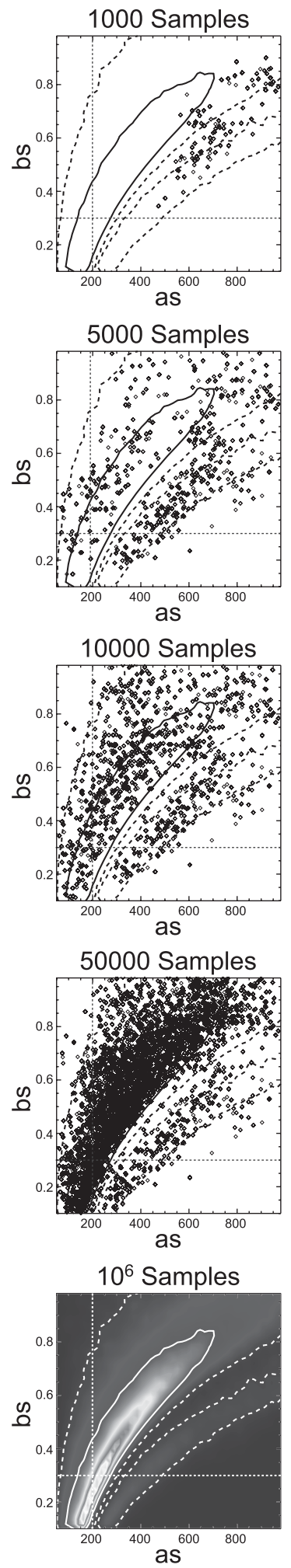

FIG. 5. Illustration of convergence of MCMC to sampling a stationary distribution for progressively greater numbers of samples. In each plot, the dashed and solid curves enclose $95 \%$ and $68 \%$ of the probability mass of the full sample, respectively. Straight solid black (white in the last plot) lines mark the location of the parameter truth value. microphysical parameters as control variables, with observations of PCP, LWP, and IWP drawn from the truth state at 30-min intervals starting at $30 \mathrm{~min}$ of simulation time and ending at $150 \mathrm{~min}$. Marginal PDFs of each cloud microphysical parameter provide an initial sensitivity estimate; because the prior PDF is uniform bounded by parameter allowable ranges, the departure of the marginal from uniform is indicative of the influence of the parameter on the model state. The shape of the marginal PDFs is consistent with the expected behavior of the model. Because fall speed parameters influence the collection process, we expect them to have an effect on the partition between liquid and ice and (especially in the stratiform region) on the precipitation rate. It is, therefore, not surprising that they exert a relatively large effect on the solution, and, consequently, produce PDFs with a well-defined mode. Density and slope intercept, on the other hand, determine the number of particles for a given particle diameter [recall Eqs. (3) and (4)]; as they decrease, the number of large particles increases and vice versa. At extremely small values of $N_{0 x}$ and $\rho_{x}$, there is a proliferation of large particles, and hence a given change in these parameters has a relatively greater effect on simulated clouds and precipitation at small parameter values than a similar change at high values.

Nonlinearity in the relationship between changes to parameter values and model output is reflected in the fact that PDFs of $a_{s}, b_{s}$, and $a_{g}$ exhibit skewness, whereas $q_{c_{0}}$ and $b_{g}$ appear to be at least bimodal. In addition to the PDFs in Fig. 6, we have also plotted the location of the true parameter values (the values used in the simulation from which observations are drawn). The fact that the specified true values do not correspond to the location of the mode [traditionally the maximum a posteriori (MAP) estimate] in several of the parameter marginal PDFs reveals the presence of multiple modes in the multidimensional joint PDF. These modes are hidden when the full PDF is integrated to produce the marginals and reflect the fact that multiple combinations of parameter values produce nearly identical model output.

An examination of the joint PDF for each possible parameter pair (Fig. 7) reveals additional information about the parameter interrelationships, as well as some of the characteristics of the multiple modes in the parameter space that were integrated out in computing the onedimensional marginal PDFs. There appears to be a direct relationship between $a_{s}$ and $b_{s}$ and between $a_{g}$ and $b_{g}$, and we note that there is nonuniqueness in the relationship between these parameters and the model output reflected in the existence of a visible secondary mode. There are also clearly discernible relationship between other parameter pairs, notably $\rho_{s}$ and $\rho_{g}, N_{0 g}$ and $\rho_{g}$, and $N_{0 s}$ and $\rho_{g}$. Here, $N_{0 r}$ and $q_{c_{0}}$ display an inverse relationship 

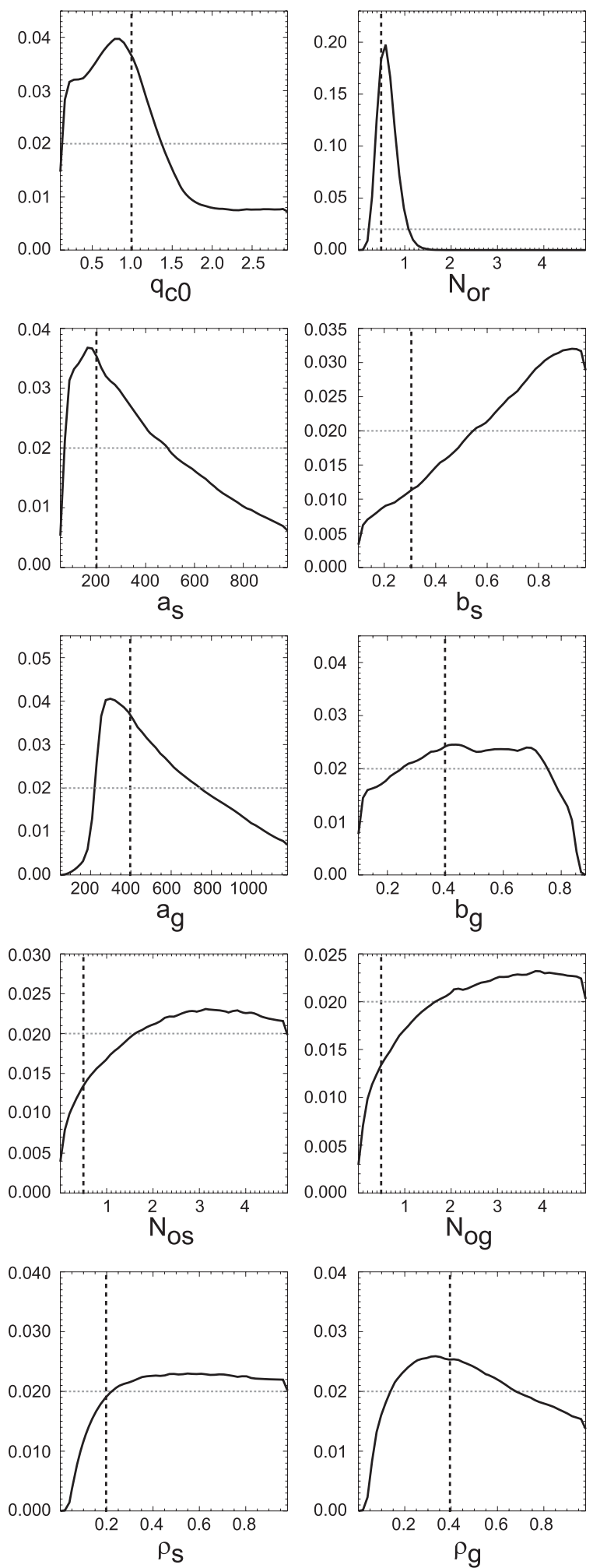

FIG. 6. Marginal PDF of each parameter for the case in which observations of PCP, LWP, and IWP are used in the inversion. In each plot, the thin horizontal dashed line depicts the probability associated with a uniform probability distribution, whereas the heavy vertical dashed line marks the location of the parameter truth value. consistent with a trade-off between the two parameters; as $N_{0 r}$ decreases, the number of large raindrops increases, and a smaller autoconversion threshold is needed to maintain the same precipitation rate. As in the onedimensional marginal PDFs (Fig. 6), many of the modes in the two-dimensional marginal PDFs appear to be located at values that are not equal to the true parameter values. We shall show that this also reflects the existence of multiple modes (nonuniqueness) hidden in the integration over the full PDF. It is interesting to note that the characteristics of the PDFs for many of the parameters, for which the marginal PDFs indicated little influence on the solution, change depending on which parameter they were paired with (specifically, $N_{0 s}, \rho_{s}$, and $N_{0 g}$ ).

We may draw the following conclusions about the sensitivity of simulated cloud liquid and ice water path and precipitation rate to changes in the values of cloud microphysical parameters. First, all parameters exert some degree of influence on the simulation of clouds and precipitation, as none of the marginal PDFs conform to a uniform distribution. Second, monotonic nonlinearity in the relationship between changes in parameter value and changes in clouds and precipitation causes changes in the degree of influence of a parameter on the solution across the range of parameter values. This was especially apparent for $\rho_{s}, N_{0 s}$, and $N_{0 g}$, which exhibit steep gradients in probability mass at low values and nearly flat distributions at relatively high values. Third, there is nonuniqueness in the relationship between parameters and simulated clouds and precipitation (nonmonotonic nonlinearity) as reflected in the presence of multiple modes in the solution. One implication of the presence of nonmonotonic nonlinearity is that data assimilation methods may have difficulty constraining cloud microphysical parameters using cloud and precipitation observations alone (VP08). The fact that a relationship exists between many pairs of parameters also provides an indication that there may not be as many degrees of freedom as the number of parameters might at first suggest.

\section{b. Sensitivity of radiative fluxes to changes in cloud microphysical parameters}

It is clear from the results presented above that cloud microphysical parameters have a nonunique relationship with simulated precipitation rate, LWP, and IWP. In the case of several of the parameters, this leads to an apparent mode in the marginal PDF that differs from the true parameter value. In this section, we examine the effect of adding observations of OLR and OSR (at each of the observing times previously used for PCP, LWP, and IWP) to the MCMC inversion, and we look for whether the increase in observation information serves to produce a 1:1 mapping between parameters and 

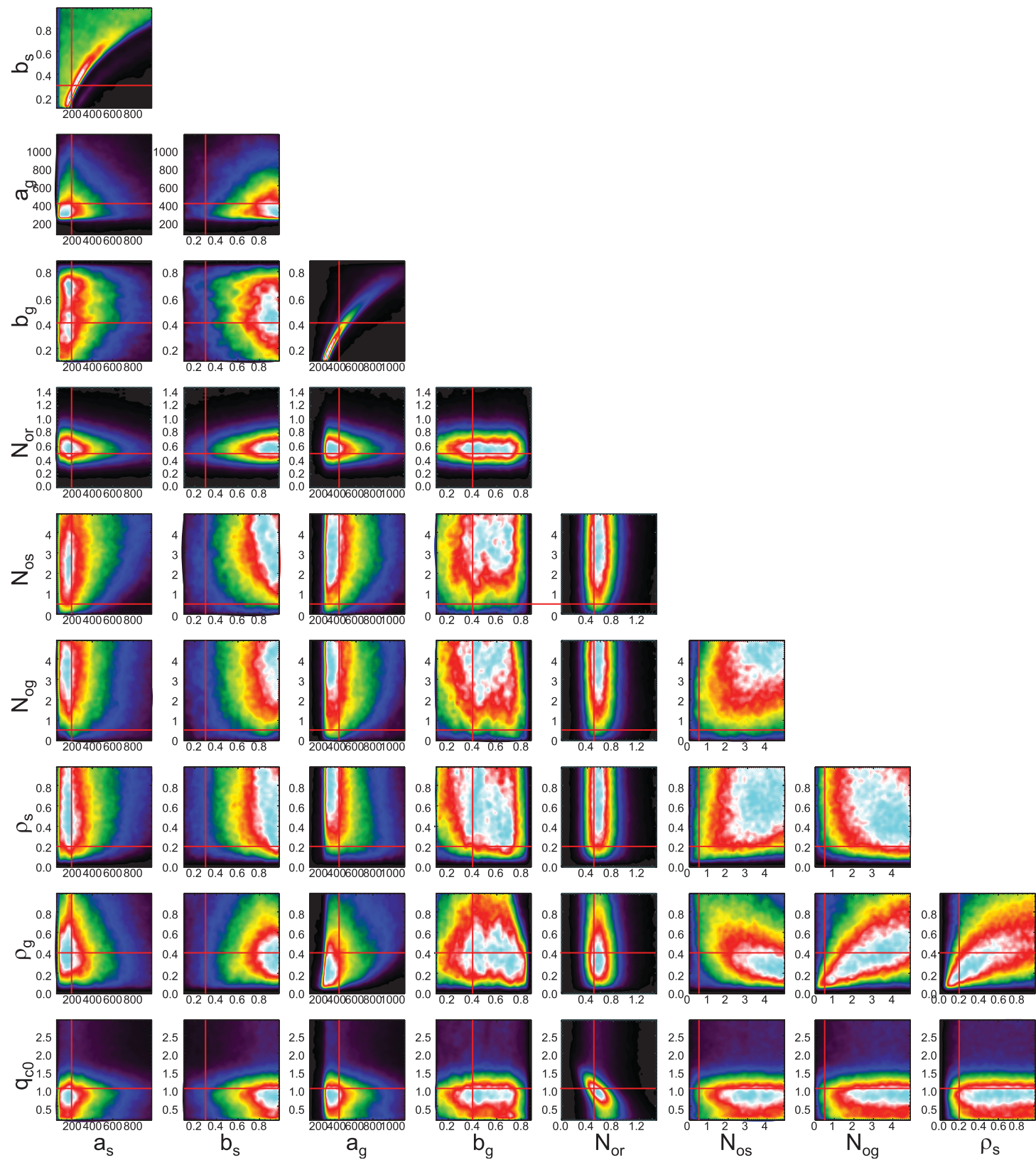

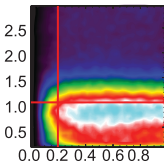

$\rho_{\mathrm{s}}$

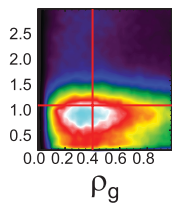

FIG. 7. Joint PDFs of each pair of parameters for the case in which observations of PCP, LWP, and IWP are used in the inversion. In each plot, dark colors represent regions of relatively low probability density, whereas lighter and warmer colors represent regions of relatively high probability density. The solid red lines mark the locations of the parameter truth values.

model output. As with precipitation rate, LWP, and IWP, top-of-atmosphere radiative fluxes are readily available from current observing systems and are highly relevant to a discussion of cloud feedbacks in the climate system. Adding OLR, OSR, or both to the observations used in the MCMC inversion also allows us to assess the sensitivity of changes in OLR and OSR to changes in parameters, via comparison with PDFs obtained from observing precipitation rate, LWP, and IWP.
The implementation of additional observations in MCMC is a straightforward matter of adding terms to the objective (cost) function. If we assume that observation uncertainties are uncorrelated, then the addition of information from new observations can be thought of as the successive conjunction of observation PDFs with the existing sample. The addition of OLR information has the largest effect on PDFs of $\rho_{s}, b_{g}, b_{s}, N_{0 s}$, and $a_{g}$ (Fig. 8). Changes in the marginal PDFs that result from 

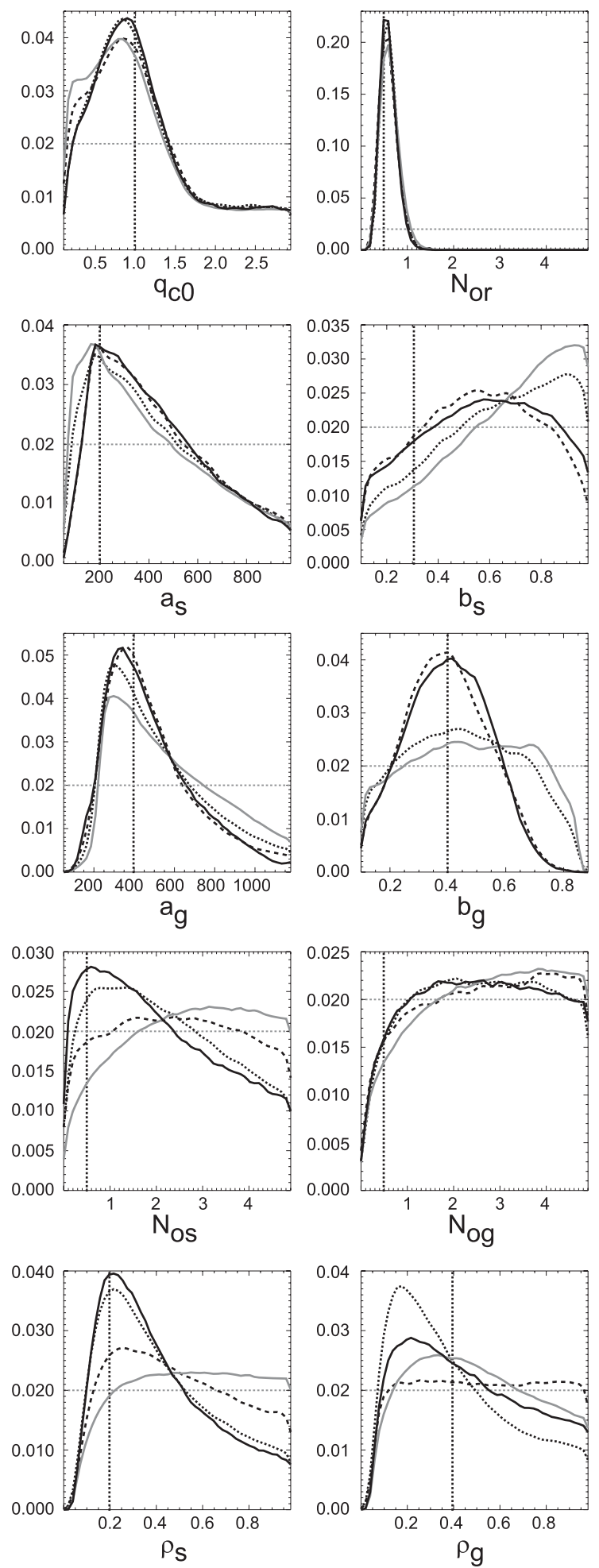

FIG. 8. Marginal PDF of each parameter for the case in which observations of PCP, LWP, and IWP are used in the inversion (solid gray line), observations of LW flux are added (dashed black line), observations of SW flux are added (dotted black line), and observations of both LW and SW are added (solid black line). As in Fig. 6, the thin horizontal dashed line depicts the probability associated with a uniform probability distribution, whereas the heavy vertical dashed line marks the location of the parameter truth value. the addition of OLR observations are consistent with the role of the parameters in the longwave portion of the radiative transfer scheme. Effective radius and optical depths are sensitive to the details of the particle size distribution, which is determined by specification of $\rho_{x}$ and $N_{0 x}$, whereas changes to the fall velocity parameters affect particle settling; vertical redistribution of ice mass changes the regions of LW absorption/emission and affects the longwave fluxes at the top of the model.

The addition of OSR observations has the greatest effect on changes in the parameters that define the snow particle size distribution $\rho_{s}$ and $N_{0 s}$. This is consistent with the fact that the cloud produced by the model is largely opaque in the visible. Consequently, changes in the lightice species (snow and pristine ice) have a greater effect on the shortwave radiation, because they are concentrated higher in the cloud. The fact that, with the addition of OSR observations, $\rho_{g}$ is approximately equal to $\rho_{s}$ may reflect the fact that, when $\rho_{g}$ is approximately equal to $\rho_{s}$ (because the true value of $N_{0 s}$ is equal to the true value of $N_{0 g}$ ), graupel at the top of the cloud appears radiatively similar to snow. This result suggests that, apart from the specification of the parameters that define the particle size distribution and fall velocity, the radiative transfer scheme does not distinguish between snow and graupel. We shall return to this later.

Unsurprisingly, because observations were assumed to be independent, the addition of information from both OLR and OSR has the effect of producing PDFs that look like a conjunction of the PDFs produced by observations of precipitation, LWP, and IWP with PDFs associated with independent observations of OLR and OSR. With all five observations included in the inversion, the primary mode in many of the parameter PDFs is now located at or close to the true parameter value. Further insight into the influence of the information contained in OLR and OSR on the parameter PDF can be seen in plots of the twodimensional margial PDFs of pairs of parameters (Fig. 9). The monotonic relationship between $a_{g}$ and $b_{g}$ and between $a_{s}$ and $b_{s}$ remains, but much of the secondary mode in $b_{s}$ that was associated with large values of $b_{s}$ is now gone.

Note the existence of a clear secondary mode in the joint PDF of $a_{g}$ with $b_{g}$ at values of $a_{g} \approx 200$ and $b_{g} \approx 0.3$, which appears to be related to a value of $\rho_{g} \approx 0.2$ in the plot of the joint PDF of $a_{g}$ and $\rho_{g}$. The fact that these values are identical to the specified true values of $a_{s}, b_{s}$, and $\rho_{s}$, respectively, is a strong indication that the model is capable of interchanging snow with graupel and producing identical output. This is partly a consequence of the fact that, aside from the parameters that define the particle size distribution, the radiation scheme does not differentiate between snow and graupel in its computation of ice optical depth; for example, there is no 

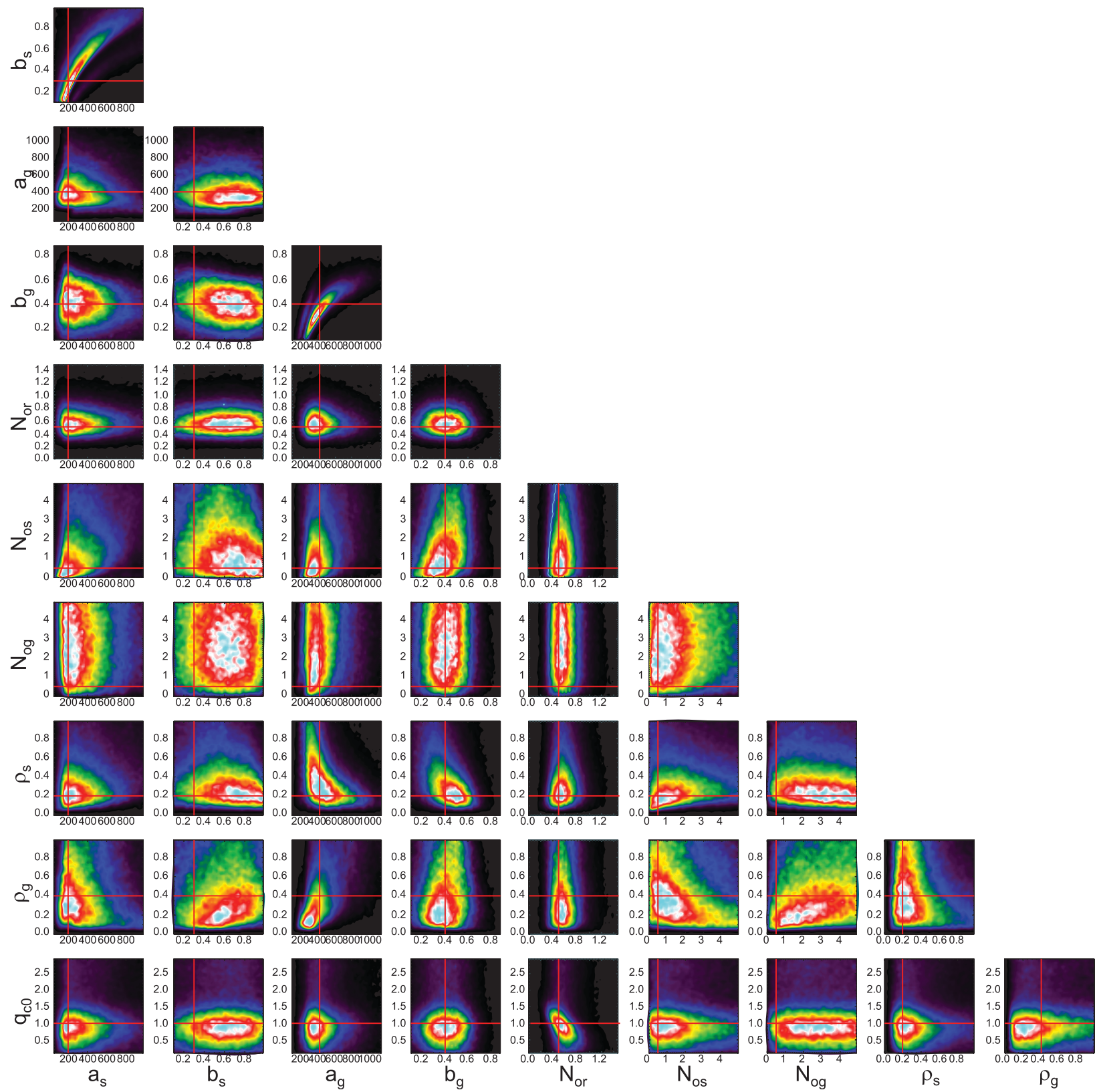

FIG. 9. As in Fig. 7, but for the case in which observations of PCP, LWP, IWP, OLR, and OSR are used in the MCMC inversion.

consideration of crystal shape in the visible scattering routines. Controlling this source of nonuniqueness in the relationship between parameters and model output requires knowledge of the physical relationships between parameters and model output.

\section{c. Functional relationship between parameters and state}

MCMC not only returns a sample of the joint parameter probability distributions, but it also includes information on the relationship between parameters and output variables via the joint PDF of model output state variables and input parameters $P[\mathbf{x}, F(\mathbf{x})]$. Examination of this PDF lends insight into a number of important properties of the model and observations. First and foremost, the PDF contains information on the functional mapping of changes in parameter values into observation space, giving insight into how model parameters affect simulation output when multiple parameters are varied. It also allows us to examine the influence of different observations on the parameter PDF at different model output times and to determine which observations should 


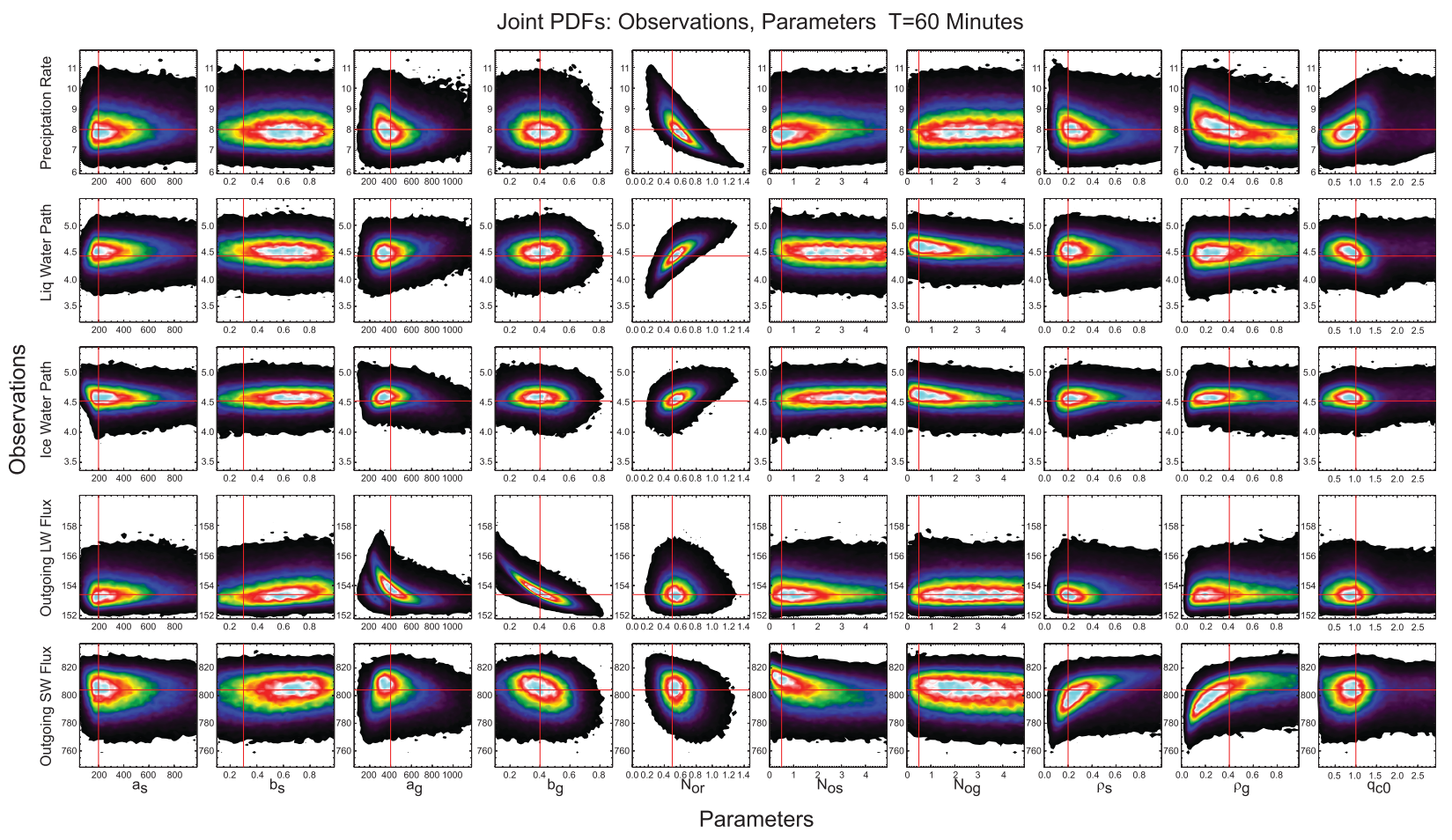

FIG. 10. Joint PDFs of parameters and model output variables for a time during the convective phase of squall-line development (60 min into the simulation). Each row corresponds to a different model state variable, whereas each column corresponds to a different model physics parameter. As in Figs. 7 and 9, solid red lines mark the locations of the observation and parameter true values.

be better constrained to eliminate secondary modes in the solution. The simulation employed in our experiments contains both a discrete convective and stratiform morphology, and it provides us with an opportunity to examine how parameter-state relationships differ in different physical environments.

\section{1) JOINT PARAMETER-STATE PDFS: CONVECTIVE}

Comparison of parameter-parameter PDFs (Fig. 9) with the joint state-parameter PDFs during the convective phase of development (Fig. 10) reveals a number of interesting features. First, we note that, although the PDF of $N_{0 g}$ is nearly uniformly distributed when $N_{0 g}$ is plotted jointly with any other parameter (Fig. 9), $N_{0 g}$ exhibits a well-defined mode around the true parameter value when plotted jointly with LWP and IWP at the convective time. The mode is biased high when $N_{0 g}$ is plotted jointly with the precipitation rate, OLR, and OSR, indicating the secondary mode is somehow associated with these state variables. Here, $N_{0 s}$ exhibits similar behavior but is well constrained when plotted versus precipitation rate, OLR, and OSR, and it exhibits a secondary mode when plotted jointly with LWP and IWP. These relationships are consistent with the observations made in the discussion of parameter-parameter joint PDFs in section $4 \mathrm{~b}$; cloud liquid and ice content are more sensitive to changes in the graupel particle size distribution, whereas radiative fluxes are more sensitive to snow. Note that the secondary mode in $a_{g}$, which can be seen in plots of the joint PDF of $a_{g}$ with $b_{g}$ (Fig. 9), is only evident when $a_{g}$ is plotted jointly with OLR. We shall explore more of the implications of this a bit later in the study. Interestingly, the only parameter for which the mode is located far from the true value when plotted with any of the observations is $b_{s}$. We have seen that $b_{s}$ and $a_{s}$ are strongly related, and it is plausible that if $a_{s}$ can be constrained to lie close to its true value, then we may expect $b_{s}$ to follow suit.

Functional relationships between parameters and state are also revealed in these plots. The clearly discernible relationships between $N_{0 r}$ and precipitation rate, LWP, and IWP are consistent with the construction of the microphysics scheme; precipitation rate monotonically decreases with an increase in $N_{0 r}$, consistent with an increase in the number of small raindrops per unit mass. Consistent with an increase in suspended liquid condensate, LWP increases with an increase in $N_{0 r}$, as does IWP; greater amounts of suspended liquid are available for conversion to snow and graupel, as well as migration to pristine ice through the Bergeron process. There is also a clear relationship between $a_{g}$ and $b_{g}$ and OLR: as $a_{g}$ and $b_{g}$ increase, OLR decreases. Here, $a_{g}$ 

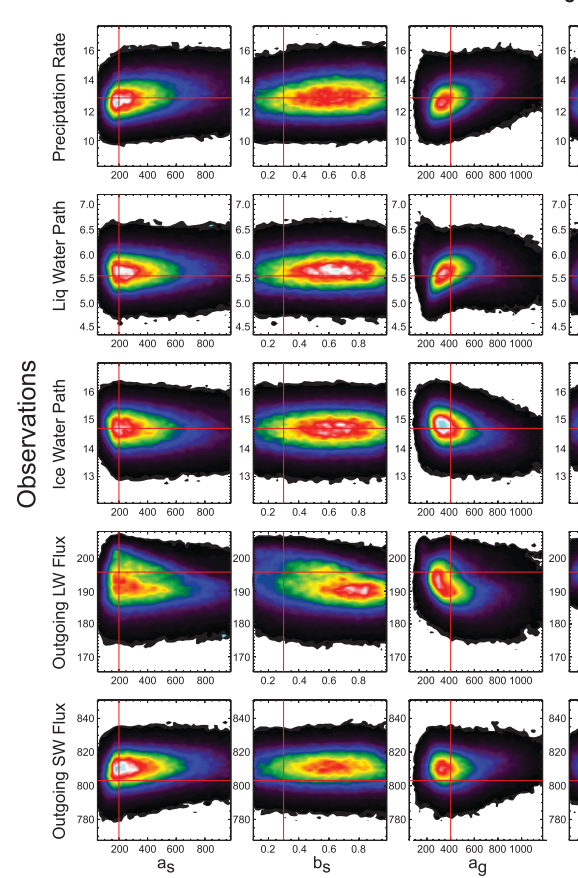

Joint PDFs: Observations, Parameters $T=120$ Minutes
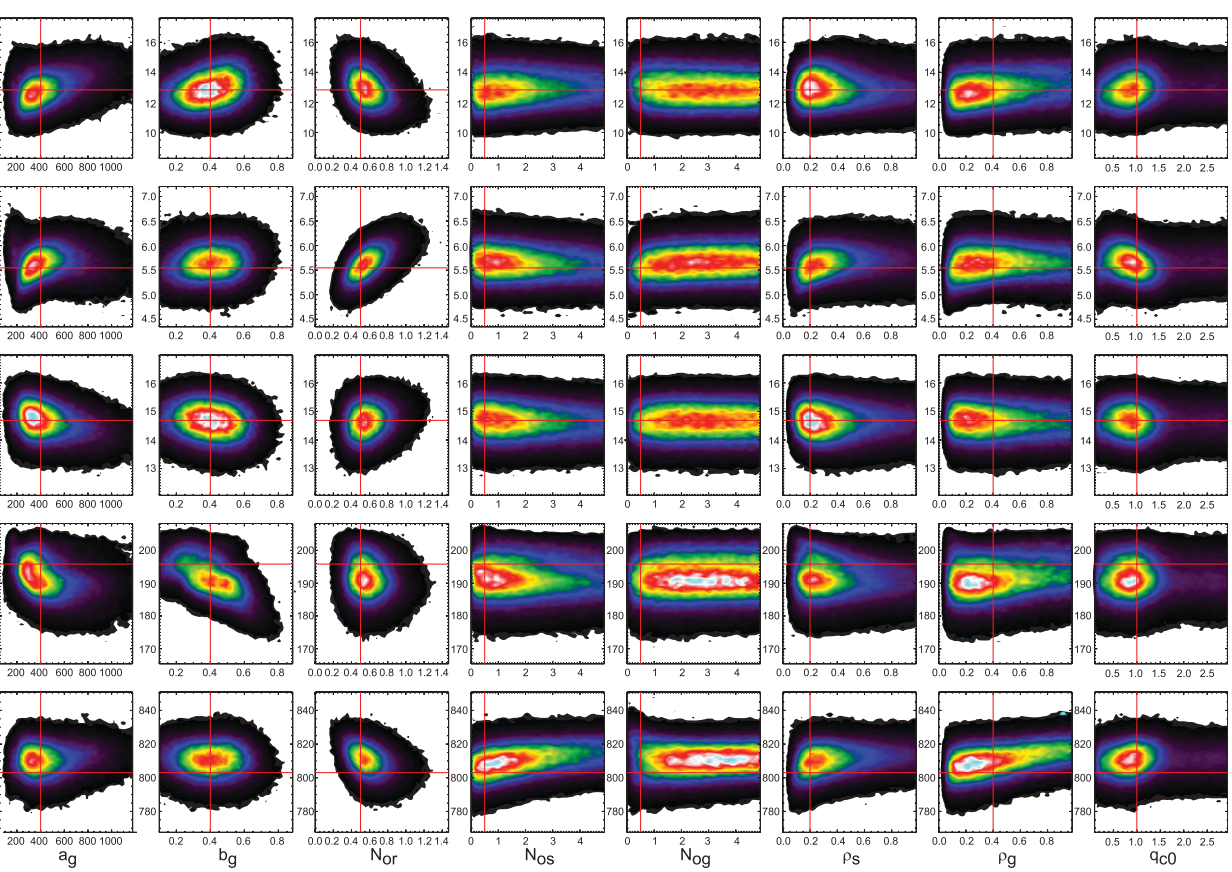

FIG. 11. As in Fig. 10, but for a time during the stratiform phase of squall-line development (120 min into the simulation).

and $b_{g}$ are proportional to the condensate fall velocity as a function of diameter and graupel particle fall speed increases with increasing $a_{g}$ and $b_{g}$. With an increase in $a_{g}$ and $b_{g}$, a greater amount of graupel settles into lower portions of the cloud, increasing the lower to middle tropospheric optical depth and reducing the OLR. There is also a clear relationship between the snow and graupel density and precipitation and OSR. The snow and graupel density primarily show up in the calculation of the slope parameter of the particle size distribution [Eq. (4)]. As density decreases, the slope of the particle size distribution decreases and there is a more rapid decrease in the number of graupel particles with increasing particle diameter-more small particles. A decrease in particle size equates to a decrease in particle velocity, an increase in suspended condensate, and a decrease in precipitation. This increase in the number of small particles also translates to greater cloud albedo and hence larger OSR. A similar effect can be observed for snow; however, the effect on the precipitation is not as strong. It is interesting to note that, compared with other model output variables, OSR exhibits a wide range of values (relative to its specified uncertainty) when paired with all of the control parameters. This not only indicates sensitivity of the shortwave radiative fluxes to changes in parameter values, but it also suggests that parameter values might be better constrained if more accurate observations of OSR were available at the convective time.

\section{2) JOINT PARAMETER OBSERVATION PDFS: STRATIFORM}

We now examine the parameter observation joint PDFs for a time in the simulation characterized by stratiform precipitation (Fig. 11). Comparison with the results obtained during the convective time period (Fig. 10) reveals that the functional relationships between parameters that directly affect the warm-rain process $\left(N_{0 r}\right.$ and $\left.q_{c_{0}}\right)$ are unchanged; an increase in $N_{0 r}$ is associated with an increase in LWP and a decrease in precipitation, whereas an increase in the autoconversion threshold is associated with a decrease in LWP and an increase in precipitation rate. The fact that the PDF of $N_{0 r}$ is broader for the stratiform time relative to the convective time is a reflection of the increasing importance of ice processes in generating stratiform precipitation, as is the increased range of model state values for a given change in ice microphysical parameters. The influence of graupel on stratiform precipitation can be seen in the fact that an increase in $a_{g}$ and $b_{g}$ (which are directly related to graupel particle fall speed) produces an increase in the precipitation rate and an attendant increase in LWP. The sensitivity of OLR to the total column-integrated water mass is reflected in the fact that the change in OLR decreases just when the change in LWP levels off. Consistent with what was observed at the convective time, OSR is sensitive to the characteristics of the snow size distribution. However, in 
contrast to the convective PDFs, an increase in both $N_{0 s}$ and $\rho_{s}$ leads to an increase in OSR. This relationship is consistent with an increase in the number of small particles near the top of the cloud producing an increase in reflected shortwave radiation. It is interesting to note that, in contrast to the convective time, changes in parameter values produce a much larger change in OLR at the stratiform time. Because the range of response in OSR is of the same order of magnitude at both times, it appears that an increase in the accuracy of observations of OLR at the stratiform time may serve to help constrain the values of key parameters. In particular, note the functional relationship between OLR and $b_{s}$ and that the secondary mode in $b_{s}$ is most evident in the joint PDF with OLR. In addition, there is a clear (though clearly nonlinear) relationship between OLR and both $a_{g}$ and $b_{g}$.

The comparison of results from convective and stratiform times allows us to distinguish between parameters that have a consistent influence on simulation output versus those whose influence is more situational. The functional relationships appear to be consistent between state variables and the parameters that define the liquid microphysics, whereas relationships between observations and parameters that define the ice particle fall speeds and size distribution change depending on when they are observed.

\section{d. Improving parameter identifiabilty by eliminating nonuniqueness}

Plots of parameter-state functional relationships immediately call to mind the notion of parameter identifiability (Anderson 2001; Aksoy et al. 2006; Tong and Xue 2008), traditionally a measure of the linear relationship between model parameters and the state, as expressed in a measure of correlation. The joint parameter-state PDFs reflect a low to moderate degree of nonlinearity for most of the parameters considered here, with the exception of $b_{s}, a_{g}, N_{0 s}$, and $N_{0 g}$, which exhibit multimodality and consequently nonmonotonic nonlinearity. This nonmonotonicity is a concern for data assimilation methods that rely on estimates of parameterparameter and parameter-observation covariance to function properly. In such cases, the combination of assumed linearity and potentially large perturbations to parameters can lead to difficulty converging to a solution. As was mentioned earlier, a nonmonotonic relationship between parameters and observations can also cause problems for MCMC algorithms that use parameterparameter covariance to generate perturbations to parameter values. In this section, we seek to eliminate nonmonotonicity in parameter-state relationships by increasing the information in key observations, and also by implementing additional constraints on parameter values consistent with our knowledge of the physical system.

As stated earlier, when observations of precipitation rate, LWP, and IWP are used in the inversion, parameter joint PDFs exhibit significant nonuniqueness; some of the joint PDFs are bimodal, whereas others exhibit multimodality in the form of a mode that is separate from the true parameter values (Figs. 7 and 12a-d). When observations of visible and infrared radiative fluxes are added to the inversion, many of the secondary modes disappear (Figs. 9 and 12e-h); however, multimodality remains, especially in many of the ice microphysical parameters. The examination of the relationship between parameter values and model output state variables at convective (Fig. 10) and stratiform (Fig. 11) time periods reveals a clear functional relationship between OLR and OSR and parameters whose PDFs continue to exhibit multiple modes. In particular, there appears to be a secondary mode in the posterior PDF of OLR centered near a value of $190 \mathrm{~W} \mathrm{~m}^{-2}$ that is closely associated with the secondary mode of many of the parameter PDFs. It follows that a natural next step is to constrain the solution space using smaller errors on the radiative fluxes to determine whether we might yet arrive at a single-mode solution for all parameters. To this end, we rerun the MCMC experiments and apply errors of $5 \mathrm{~W} \mathrm{~m}^{-2}$ on OLR and OSR; these correspond to $50 \%$ and $25 \%$ of the previously assumed errors, respectively (Table 2), and they are consistent with a reasonable increase in accuracy for the next generation of spaceborne sensors (T. L'Ecuyer 2009, personal communication). In the interest of brevity, we focus on those ice microphysical parameters whose PDFs exhibit both multimodality and a functional relationship with OLR and OSR.

The parameter joint PDFs that correspond to observations of precipitation rate, LWP, and IWP alone are plotted in Figs. 12a-d, those that correspond to the addition of information from OLR and OSR with large assumed error are plotted in Figs. 12e-h, and PDFs that result from increasing observation accuracy on radiative fluxes are presented in Figs. 12i-l. In contrast to mitigating parameter nonuniqueness, the addition of observation information has actually enhanced it. This is because the nonuniqueness in the solution is a product of the underlying model formulation and is not associated with the particular choice of observations. As was mentioned earlier, there is no consideration of the distinction between snow and graupel in the radiative transfer scheme, apart from the differences in their assumed particle size distribution. This leads to inherent nonuniqueness in the relationship between microphysical parameters and 

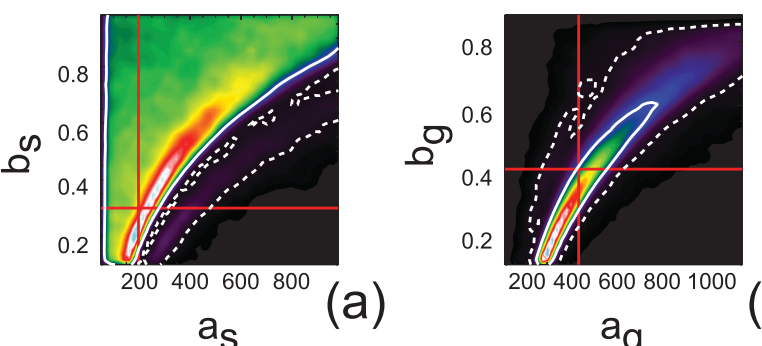

(a)
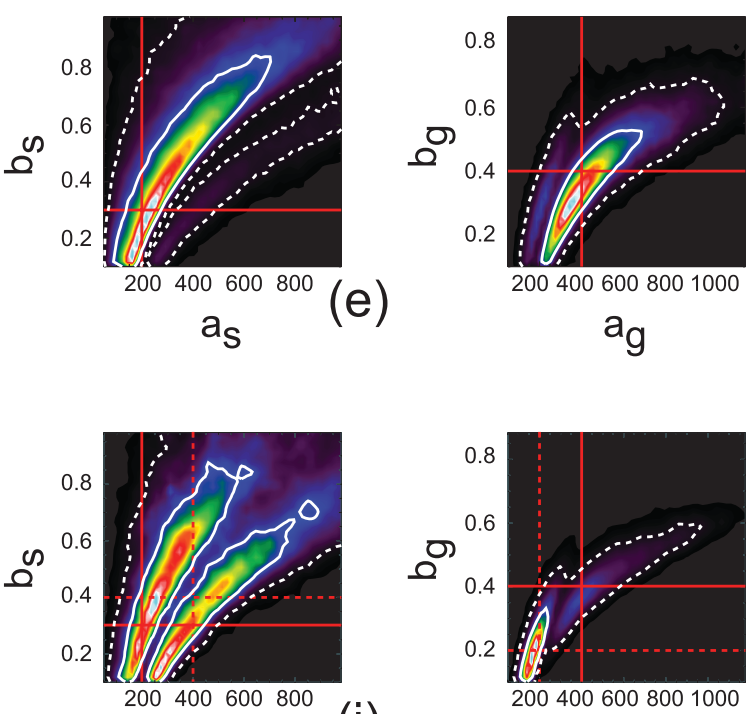

$a_{S}$

(i)

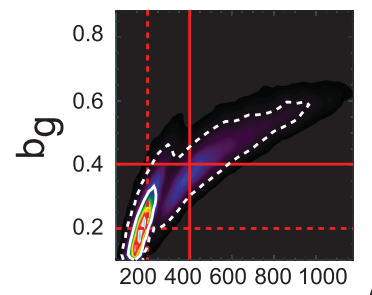

$a_{g}$

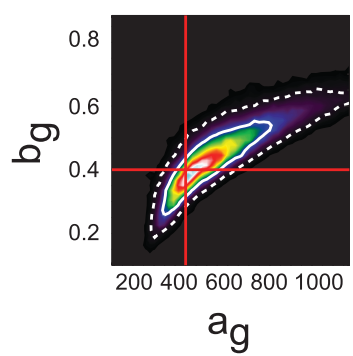

(f)

(b)

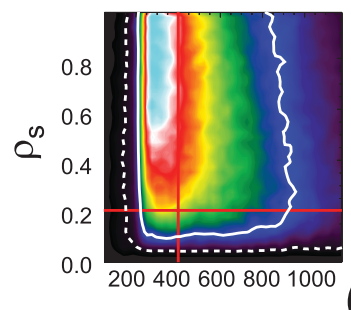

ag

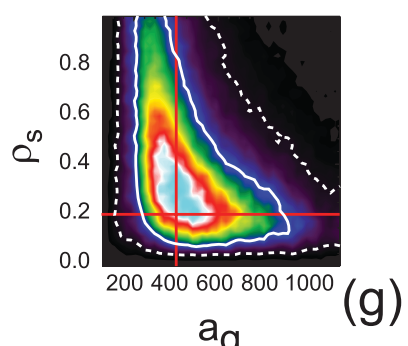

$\mathrm{a}_{\mathrm{g}}$

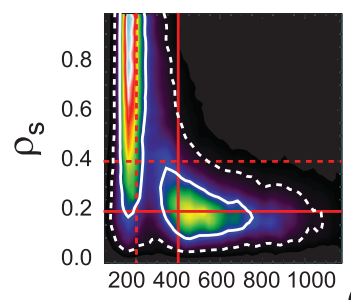

$\mathrm{a}_{\mathrm{g}}$

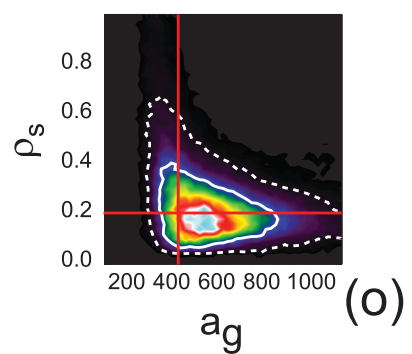

(c)

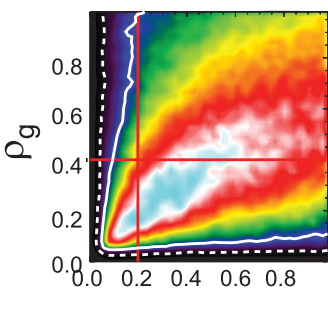

$\rho_{S}$

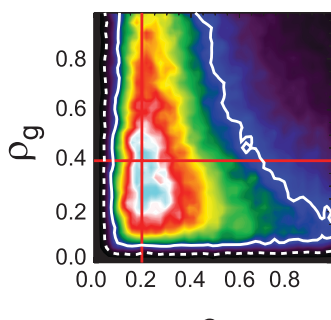

$\rho_{\mathrm{s}}$

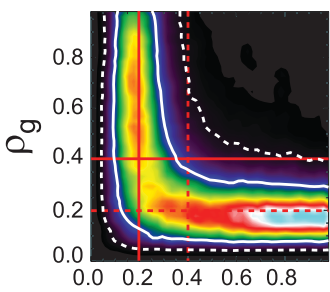

$\rho_{S}$

(l)

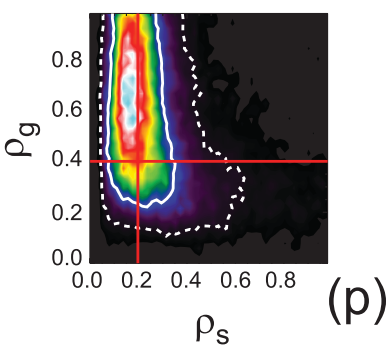

FIG. 12. Plots of select joint PDFs for observations of PCP, LWP, and IWP (PLI; row 1), PLI plus observations of OLR and OSR (PLIOO; row 2), PLIOO with reduced error on the radiative fluxes (row 3), and PLIOO with reduced error on the radiative fluxes and the constraint that the graupel fall speed coefficient exceed the snowfall speed coefficient $\left(a_{g}>a_{s}\right)$. White solid and dashed curves correspond to the $68 \%$ and $95 \%$ probability contours, respectively; red solid lines indicate the position of the parameter truth value; and red dashed lines indicate the truth value for the situation in which snow and graupel parameters are interchanged.

simulated radiative fluxes. This is reflected in the fact that two modes in each of the parameter joint PDFs pass through the truth values of both snow and graupel (we have highlighted this by plotting the truth values that correspond to replacing snow parameters with graupel and vice versa on Figs. 12i-1). Note also that, although the primary mode in the parameter joint PDFs generally intersects the truth values for large OLR and OSR uncertainty, when observation accuracy is increased, the primary modes are now far removed from the truth for all but $a_{s}$ and $b_{s}$, for which there appears to be approximately equal mass in each mode. This shift in mass is preferably from the true graupel values toward the true snow values, and it likely results from the relatively larger increase in SW flux information relative to LW flux; the shortwave flux is more sensitive to the characteristics of the snow particle size distribution, and hence it constrains the graupel particle size distribution to look like snow. 
The results are indicative of the problems inherent in the estimation of nonunique parameters. With relatively large observation error, nonuniqueness can be hidden by the relatively large variance around each mode in the PDF. In practical terms, minimization techniques that rely on gradients will be more likely to become localized within one mode or the other when observation uncertainty is reduced. Our results demonstrate that the addition of information can actually lead to bias in the solution rather than to an improved parameter estimate. If we desire a 1:1 mapping between parameters and model output state, then we have essentially three options: change the observing system (such that parameters and observations have a 1:1 relationship), modify the model to eliminate the source of nonuniqueness, or modify the construction of the inverse method. Let us assume (as is the case for modern data assimilation and observing systems) that model development is an ongoing but slow process, and that we are already using all of the available observations. It remains, then, to transform the current model-state relationship into one that provides a 1:1 mapping from parameters to model output without modifying the model or adding additional observations. This can be done by introducing additional knowledge of the physical system into the construction of the inverse method. In the case of the current three-ice-class microphysics scheme, this consists of information that will allow the inverse method to discriminate between aspects of the snow and graupel particle size distribution so that the joint PDF of the ice microphysical parameters is rendered unimodal. We employ the knowledge that graupel particles generally fall faster than snow and apply the constraint that $a_{s}<a_{g}$. This additional constraint is easily incorporated in MCMC via an additional reject criterion; if the algorithm samples a region of the parameter space (randomly generates a set of parameters) for which $a_{s} \geq a_{g}$, then that parameter set is immediately rejected.

Results of applying a constraint on $a_{s}$ and $a_{g}$ are plotted in Figs. $12 \mathrm{~m}-\mathrm{p}$ and clearly show the effectiveness of applying this modification to the MCMC inversion. Not only are the secondary modes in the joint PDFs of $a_{s}, b_{s}$ and $a_{g}, b_{g}$ eliminated, but the snow and graupel density parameters also lose their second modes. It is interesting to compare the parameter joint PDFs through the progressive addition of observation information. The functional form of the relationship between the fall speed parameters does not change with the addition of information from radiative fluxes; however, the relationship between $a_{g}, \rho_{s}$ and $\rho_{s}, \rho_{g}$ changes significantly. When precipitation rate, LWP, and IWP are observed in absence of radiative fluxes, there is little apparent relationship between $a_{g}$ and $\rho_{s}$ and a direct linear relationship between $\rho_{s}$ and $\rho_{g}$. Following the addition of information from radiative fluxes, $a_{g}$ and $\rho_{s}$ display an inverse relationship, whereas $\rho_{s}$ and $\rho_{g}$ appear to be uncorrelated. The assumption of increased accuracy for the observations of radiative flux reveals the apparent relationship between $a_{g}$ and $\rho_{s}$ to consist of two distinct modes in the joint space, whereas $\rho_{s}$ and $\rho_{g}$ appear to be closely related, though the relationship is nonlinear. Application of the $a_{g}>a_{s}$ constraint changes the relationship between $\rho_{s}$ and $\rho_{g}$ yet again, revealing it to have been a by-product of the nonuniqueness in the construction of the model.

\section{Summary and conclusions}

In this paper, we introduced a Lagrangian column framework for evaluating model physics parameterizations and described how nonlinear inverse methods can be used to examine the details of model physics uncertainty. We then used this framework to explore the characteristics of uncertainty due to specification of parameters in a single-moment bulk cloud microphysical parameterization. The MCMC inverse method returned a robust sample of the joint PDF of the parameters and model output variables, from which we were able to obtain information on the sensitivity of model output to changes in parameters. This information includes details of the relationship between parameters, which parameters had the greatest effect on model output, and the nature of the parameter-state functional relationship. The major findings of this study include the following:

- All of the microphysical parameters had some effect on the solution, but the effects differed depending on the model state variable examined. Parameters that define the fall velocity of snow and graupel, along with the slope intercept of the rain size distribution and the cloud-to-rain autoconversion mass mixing ratio threshold, had the greatest effect on simulated precipitation rate and cloud liquid and ice water paths. Parameters that define the graupel particle size distribution had the greatest effect on OLR, whereas parameters that define the snow particle size distribution had the greatest effect on OSR.

- Marginal PDFs tended to mask multimodality in the solution, and in some cases, a wrong estimate of parameter values could be obtained if the mode of the parameter marginal PDF was used.

- Joint PDFs of parameter pairs proved to be effective for revealing nonuniqueness (nonmonotonic nonlinearity) in the functional relationship between parameters that define the crystal shape and particle size distribution and simulated precipitation rate, LWP, IWP, and radiative fluxes. 
- Joint PDFs of parameters with model state variables returned useful information on the details of the sensitivity of model output to changes in parameters, and they indicated that the functional relationship between parameters and model output changes depending on cloud morphology.

- Reductions in observation uncertainty served to increase the definition of the discrete solutions (as represented in multiple modes in the parameter space) and to exacerbate the problem of nonuniqueness and reduced parameter identifiability.

- Knowledge of the physical nature of the system can be used to greatly reduce, or even eliminate, the potential for a multimode solution. In this case, endemic nonmonotonic nonlinearity was addressed by imposing a simple requirement that graupel particles fall faster than snow. The flexibility of the MCMC framework makes implementation of this type of constraint trivial.

Multimodality in the joint parameter PDF has implications for the decrease in parameter identifiability noted in previous studies; with increasing dimension in the parameter space, the potential for a multimode solution increases. However, it is interesting to note that the realization of multimodality in the posterior PDF depends on which parameters are included in the sample. Because of nonlinearity in parameter-parameter and parameterstate relationships, parameter identifiability does not decrease smoothly as the number of parameters included as control variables in the assimilation increase. Instead, there is likely to be a sudden decrease in parameter identifiability as the potential for nonuniqueness in the parameter-state relationship is realized.

Nonmonotonic nonlinearity in the model-state functional relationship (nonuniqueness) and associated multimode parameter joint PDF reveals potential problems for data assimilation techniques that rely on the assumption of Gaussian posterior probability distributions. As is well known, this assumption leads to mean estimates that may deviate significantly from the maximum likelihood parameter values, depending on the nature of nonlinearity in the model. Although it may be possible for ensemble-type assimilation systems to return a robust estimate of the maximum a posteriori solution through successive addition of information from observations (VP08), a key goal of ensemble prediction is to more fully span the true range of variability in the natural system. As such, eliminating viable parameter combinations simply for the sake of returning a robust estimate may unrealistically restrict the robustness of ensemble prediction systems. Our results suggest that ensemble prediction systems should represent uncertainties inherent in physical parameterizations, even if parameter estimation is not included in the data assimilation system. Ensemble forecast studies that include mixed physics ensembles demonstrate the potential benefit of this approach (Stensrud et al. 2000). Determining the optimal approach for representing uncertainties in model physics that have a nonmonotonic nonlinear relationship to observations is one of the outstanding challenges in modern data assimilation and ensemble prediction research.

The fact that parameter-state relationships can change between convective and stratiform times within the same simulation implies that the relationships between changes in parameters and changes in model output are sure to differ for different cloud system types. These are also likely to change if the microphysics is allowed to interact with the dynamics in the model. Because cloud microphysical processes in nature directly feed back to cloudscale (and larger) dynamics, we plan to run MCMC-based studies of parameter sensitivity using $2 \mathrm{D}$ and $3 \mathrm{D}$ versions of the GCE model. In addition, we note that, because the PDFs produced by the inversion are conditional and depend on the chosen model and observations, use of observations more directly related to the details of the particle size distribution (e.g., radar reflectivity) will change the nature of the solution space and may serve to render a unimodal PDF. Conversely, observations that are less directly related to cloud microphysical characteristics (e.g., wind and temperature) will likely result in an even greater degree of nonuniqueness in the parameterobservation relationship. We leave the detailed investigation of how increased (or decreased) observation information content associated with the use of different observing systems contributes to parameter identifiability for future work.

In the near future, we plan to apply the framework introduced in this paper to other cloud system types (e.g., shallow convection, airmass convection, stratus, and frontal clouds) and to explore the utility of the nonlinear inverse framework for understanding uncertainty in GCM parameterizations. We also note that the MCMC algorithm operates over the length of the simulation, requiring parameter values to be held constant. VP08 showed that the nonuniqueness in the model solution could be mitigated through sequential addition of observation information. Hence, we plan to apply the nonlinear analysis technique developed in VP08 to a subset of the microphysical parameters examined here for the purpose of examining whether the sequential update of parameter values may serve to reduce the solution to a singleparameter set.

Acknowledgments. Wei-Kuo Tao and Steve Lang assisted with interpretation of the GCE code, and Tristan L'Ecuyer provided estimates of observation 
uncertainty. Ben Shipway's 1D CRM comparison studies (available online at http://www.convection.info/ microphysics/); provided the basis for the functional forms of the vertical distribution of vertical motion and vapor mixing ratio tendency. The comments of two anonymous reviewers served to improve the clarity and readability of the text. This work was supported by the NASA MAP program under Grants NNX08AF77G, NNX09AJ46G, and NNX09AJ43G.

\section{REFERENCES}

Aksoy, A., F. Zhang, and J. W. Nielsen-Gammon, 2006: Ensemblebased simultaneous state and parameter estimation in a twodimensional sea-breeze model. Mon. Wea. Rev., 134, 2951-2970.

Anderson, J. L., 2001: An ensemble adjustment Kalman filter for data assimilation. Mon. Wea. Rev., 129, 2884-2903.

Evensen, G., 2006: Data Assimilation: The Ensemble Kalman Filter. Springer, $279 \mathrm{pp}$.

Ferrier, B. S., 1994: A double-moment multiple-phase four-class bulk ice scheme. Part I: Description. J. Atmos. Sci., 51, 249280.

_ J. Simpson, and W.-K. Tao, 1996: Factors responsible for precipitation efficiencies in midlatitude and tropical squall simulations. Mon. Wea. Rev., 124, 2100-2125.

Fovell, R. G., and Y. Ogura, 1989: Effect of vertical wind shear on numerically simulated multicell storm structure. J. Atmos. Sci., 46, 3144-3176.

— , and P.-H. Tan, 1998: The temporal behavior of numerically simulated multicell-type storms. Part II: The convective cell life cycle and cell regeneration. Mon. Wea. Rev., 126, $551-577$.

Gelman, A. G., G. O. Roberts, and W. R. Gilks, 1996: Efficient Metropolis jumping rules. Bayesian Statistics $V$, J. M. Bernardo et al., Eds., Oxford University Press, 599-608.

_ J. B. Carlin, H. S. Stern, and D. B. Rubin, 2004: Bayesian Data Analysis. 2nd ed. Chapman and Hall/CRC, 668 pp.

Gilmore, M. S., J. M. Straka, and E. N. Rasmussen, 2004: Precipitation uncertainty due to variations in precipitation particle parameters within a simple microphysics scheme. Mon. Wea. Rev., 132, 2610-2627.

Grabowski, W. W., 2003: Impact of cloud microphysics on convective-radiative quasi equilibrium revealed by cloudresolving convective parameterization. J. Climate, 16, 34633475.

— X. Wu, and M. W. Moncrieff, 1999: Cloud-resolving modeling of tropical cloud systems during phase III of GATE. Part III: Effects of cloud microphysics. J. Atmos. Sci., 56, 2384-2402.

Haario, H., E. Saksman, and J. Tamminen, 1999: Adaptive proposal distribution for random walk Metropolis algorithm. Comput. Stat., 14, 375-395.

Heymsfield, A. J., A. Bansemer, P. R. Field, S. L. Durden, J. L. Smith, J. E. Dye, W. Hall, and C. A. Grainger, 2002: Observations and parameterizations of particle size distributions in deep tropical cirrus and stratiform precipitating clouds: Results from in situ observations in TRMM field campaigns. J. Atmos. Sci., 59, 3457-3491.

Houze, R. A., 2004: Mesoscale convective systems. Rev. Geophys., 42, RG4003, doi:10.1029/2004RG000150.

Kalnay, E., 2003: Atmospheric Modeling, Data Assimilation, and Predictability. Cambridge University Press, 341 pp.
Lang, S., W.-K. Tao, R. Cifelli, W. Olson, J. Halverson, S. Rutledge, and J. Simpson, 2007: Improving simulations of convective systems from TRMM LBA: Easterly and westerly regimes. J. Atmos. Sci., 64, 1141-1164.

L'Ecuyer, T. S., and G. L. Stephens, 2002: An estimation-based precipitation retrieval algorithm for attenuating radars. J. Appl. Meteor., 41, 272-285.

— and - 2003: The tropical oceanic energy budget from the TRMM perspective. Part I: Algorithm and uncertainties. J. Climate, 16, 1967-1985.

Lewis, J., S. Lakshmivarahan, and S. Dhall, 2006: Dynamic Data Assimilation: A Least Squares Problem. Cambridge University Press, $680 \mathrm{pp}$

Lin, Y.-L., R. D. Farley, and H. D. Orville, 1983: Bulk parameterization of the snow field in a cloud model. J. Climate Appl. Meteor., 22, 1065-1092.

Locatelli, J. D., and P. V. Hobbs, 1974: Fall speeds and masses of solid precipitation particles. J. Geophys. Res., 79, 2185-2197.

Meng, Z., and F. Zhang, 2007: Tests of an ensemble Kalman filter for mesoscale and regional-scale data assimilation. Part II: Imperfect model experiments. Mon. Wea. Rev., 135, 14031423.

Meyers, M. P., R. L. Walko, J. Y. Harrington, and W. R. Cotton, 1997: New RAMS cloud microphysics parameterization. Part II: The two-moment scheme. Atmos. Res., 45, 3-39.

Mitchell, D. L., 1996: Use of mass- and area-dimensional power laws for determining precipitation particle terminal velocities. J. Atmos. Sci., 53, 1710-1723.

Moncrieff, M. W., 1992: Organized convective systems: Archetypal dynamical models, mass and momentum flux theory, and parameterization. Quart. J. Roy. Meteor. Soc., 118, 819-850.

Mosegaard, K., and A. Tarantola, 2002: Probabilistic approach to inverse problems. International Handbook of Earthquake and Engineering Seismology: Part A, W. H. K. Lee et al., Eds., Academic Press, 237-265.

Petch, J. C., and M. E. B. Gray, 2001: Sensitivity studies using a cloud-resolving model simulation of the tropical west Pacific. Quart. J. Roy. Meteor. Soc., 127, 2287-2306.

Posselt, D. J., T. S. L'Ecuyer, and G. L. Stephens, 2008: Exploring the error characteristics of thin ice cloud property retrievals using a Markov chain Monte Carlo algorithm. J. Geophys. Res., 113, D24206, doi:10.1029/2008JD010832.

Rodgers, C. D., 2000: Inverse Methods for Atmospheric Sounding: Theory and Practice. World Scientific, 238 pp.

Roy, S. S., R. K. Datta, R. C. Bhatia, and A. K. Sharma, 2005: Dropsize distributions of tropical rain over south India. Geofizika, 22, 105-130.

Rutledge, S. A., and P. V. Hobbs, 1983: The mesoscale and microscale structure and organization of clouds and precipitation in midlatitude cyclones. VIII: A model for the "seederfeeder" process in warm-frontal rainbands. J. Atmos. Sci., 40, $1185-1206$.

— and -1984 : The mesoscale and microscale structure and organization of clouds and precipitation in midlatitude cyclones. Part XII: A diagnostic modeling study of precipitation development in narrow cold-frontal rainbands. J. Atmos. Sci., 41, 2949-2972.

Saleeby, S. M., and W. R. Cotton, 2004: A large-droplet mode and prognostic number concentration of cloud droplets in the Colorado State University Regional Atmospheric Modeling System (RAMS). Part I: Module descriptions and supercell test simulations. J. Appl. Meteor., 43, 182-195. 
Stensrud, D. J., J.-W. Bao, and T. T. Warner, 2000: Using initial condition and model physics perturbations in short-range ensemble simulations of mesoscale convective systems. Mon. Wea. Rev., 128, 2077-2107.

Tamminen, J., 2004: Validation of nonlinear inverse algorithms with Markov chain Monte Carlo method. J. Geophys. Res. 109, D19303, doi:10.1029/2004JD004927.

, and E. Kyrola, 2001: Bayesian solution for nonlinear and nonGaussian inverse problems by Markov Chain Monte Carlo method. J. Geophys. Res., 106, 14 377-14 390.

Tao, W.-K., and J. Simpson, 1993: Goddard Cumulus Ensemble Model. Part I: Model description. Terr. Atmos. Oceanic Sci., 4, 35-72.

_ J. R. Scala, B. S. Ferrier, and J. Simpson, 1995: The effect of melting processes on the development of a tropical and a midlatitude squall line. J. Atmos. Sci., 52, 1934-1948.

_- and Coauthors, 2003: Microphysics, radiation, and surface processes in the Goddard Cumulus Ensemble (GCE) model. Meteor. Atmos. Phys., 82, 97-137.

Tarantola, A., 2005: Inverse Problem Theory and Methods for Model Parameter Estimation. SIAM, 342 pp.
Tokay, A., and D. A. Short, 1996: Evidence from tropical raindrop spectra of the origin of rain from stratiform versus convective clouds. J. Appl. Meteor., 35, 355-371.

Tong, M., and M. Xue, 2008: Simultaneous estimation of microphysical parameters and atmospheric state with simulated radar data and ensemble square root Kalman filter. Part I: Sensitivity analysis and parameter identifiability. Mon. Wea. Rev., 136, 1630-1648.

van den Heever, S. C., and W. R. Cotton, 2004: The impact of hail size on simulated supercell storms. J. Atmos. Sci., 61, 1596-1609.

Vukicevic, T., and D. J. Posselt, 2008: Analysis of the impact of model nonlinearities in inverse problem solving. J. Atmos. Sci., 65, 2803-2823.

Walko, R. L., W. R. Cotton, M. P. Meyers, and J. Y. Harrington, 1995: New RAMS cloud microphysics parameterization. Part I: The single-moment scheme. Atmos. Res., 38, 29-62.

Wu, X., W. D. Hall, W. W. Grabowski, M. W. Moncrieff, W. D. Collins, and J. T. Kiehl, 1999: Long-term behavior of cloud systems in TOGA COARE and their interactions with radiative and surface processes. Part II: Effects of ice microphysics on cloud-radiation interaction. J. Atmos. Sci., 56, 3177-3195. 